Discrete Particle Noise in Particle-in-Cell Simulations of Plasma Microturbulence

W. M. Nevins, G. W. Hammett, A. M. Dimits, W. Dorland, D. E. Shumaker

September 23, 2005

The Physics of Plasmas 
This document was prepared as an account of work sponsored by an agency of the United States Government. Neither the United States Government nor the University of California nor any of their employees, makes any warranty, express or implied, or assumes any legal liability or responsibility for the accuracy, completeness, or usefulness of any information, apparatus, product, or process disclosed, or represents that its use would not infringe privately owned rights. Reference herein to any specific commercial product, process, or service by trade name, trademark, manufacturer, or otherwise, does not necessarily constitute or imply its endorsement, recommendation, or favoring by the United States Government or the University of California. The views and opinions of authors expressed herein do not necessarily state or reflect those of the United States Government or the University of California, and shall not be used for advertising or product endorsement purposes. 


\title{
Discrete particle noise in particle-in-cell simulations of plasma microturbulence
}

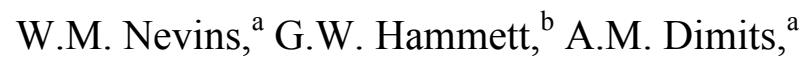 \\ W. Dorland, ${ }^{\mathrm{c}}$ and D.E. Shumaker ${ }^{\mathrm{a}}$
}

September 19, 2005

${ }^{a}$ Lawrence Livermore National Laboratory, Livermore, CA 94550

${ }^{\mathrm{b}}$ Princeton Plasma Physics Laboratory, Princeton, NJ 08536

${ }^{\text {c }}$ University of Maryland, College Park, MD 20742 


\section{Abstract}

Recent gyrokinetic simulations of electron temperature gradient (ETG) turbulence with the global particle-in-cell (PIC) code GTC [Z. Lin, et al., Proc. $20^{\text {th }}$ Fusion Energy Conference, Vilamoura, 2004 (IAEA, Vienna, 2005)] yielded different results from earlier flux-tube continuum codes simulations [F. Jenko and W. Dorland, Phys. Rev. Lett. 89, 225001 (November, 2002)] despite similar plasma parameters. Differences between the simulation results were attributed to insufficient phase-space resolution and novel physics associated with global simulation models. The results of the global PIC code are reproduced here using the flux-tube PIC code PG3EQ [A.M. Dimits, et al., Phys. Rev. Lett. 77, 71 (1996)], thereby eliminating global effects as the cause of the discrepancy. The late-time decay of ETG turbulence and the steady-state heat transport observed in these PIC simulations is shown to result from discrete particle noise. Discrete particle noise is a numerical artifact, so both these PG3EQ simulations and (by inference) the GTC simulations that they reproduced have little to say about steady-state ETG turbulence and the associated anomalous heat transport. In the course of this work several diagnostics are developed to retrospectively test whether a particular PIC simulation is dominated by discrete particle noise. 


\section{Introduction}

Particle-in-cell (PIC) simulations are one of several computational techniques that have been useful in plasma physics research. As with all numerical algorithms, attention must be paid to convergence issues. In the PIC approach, these issues include discrete particle noise and convergence with the number of particles. Discrete particle effects in plasmas are associated with collisions, and generally decrease with increasing particle number. It is impractical to include as many particles in PIC simulation codes as are present in typical experimental plasmas, so PIC simulation codes seek to reduce discrete particle "noise" through the use of grids, smoothing (which is introduced while solving for the electro-magnetic fields), and finite-size particles. In addition, modern plasma microturbulence codes further reduce discrete particle effects by representing only the

(small) departure of the particle distribution function from a Maxwellian. ${ }^{1-5} \mathrm{~A}$ consequence of these noise reduction techniques is that the discrete particle effects which remain do not accurately reproduce collisional effects in the plasma being modeled, and must be viewed as numerical artifacts of the PIC (Monte-Carlo-like) integration technique. If the discrete particle noise does become important in a PIC simulation, then that simulation cannot be viewed as a faithful model of plasma microturbulence as it occurs in experimental plasmas.

Discrete particle noise is not just a notional problem in PIC simulations of plasma microturbulence. The work reported here began as an effort to understand the very 
different levels of heat transport reported in continuum simulations ${ }^{6-8}$ (which used finitedifference and/or spectral algorithms) and simulations employing the global gyrokinetic PIC code GTC $^{9-11}$ of electron temperature gradient (ETG) turbulence at nearly the same plasma parameters. The plasma parameters in question were originally used in the Cyclone ion temperature gradient (ITG) turbulence benchmarking effort, ${ }^{12}$ so we will refer to this case as Cyclone base-case-like ETG turbulence. These very different simulation results have been variously attributed to insufficient phase-space resolution and/or an insufficient simulation volume $e^{9-11}$ in the simulations reported in Refs. 6-8, and to novel physics associated with toroidicity and/or global simulation models. ${ }^{9-11}$

To investigate these claims, we carried out independent PIC simulations of Cyclone base-case-like ETG turbulence using the flux-tube PIC code, PG3EQ. ${ }^{13}$ We have reproduced important features of the GTC simulations, ${ }^{9-11}$ including the shift to longer wavelength after the initial saturation of the linear ETG instability, the late-time decay of the ETG turbulence, and the relatively low steady-state heat transport, thereby eliminating global effects as the source of the discrepancy between the ETG turbulence reported in Refs. 6-8 and Refs. 9-11. However, we will demonstrate that both the latetime decay of the ETG turbulence and the steady-state heat transport observed in our PG3EQ simulations and, by inference, in the GTC simulations reported in Refs. 9-11 is a consequence of discrete particle noise. Hence, the PG3EQ simulations reported here and the GTC simulations reported in Refs. 9-11 have little to say about steady-state heat transport associated with ETG turbulence in experimental plasmas. High-fidelity simulations of ETG turbulence with PIC codes will require even more particles than have 
been used to date, and/or further effort to develop and implement additional noise reduction techniques in PIC codes. ${ }^{14-20}$

These rather surprising results with respect to PIC simulations of ETG turbulence led us to revisit our analysis of Cyclone base-case ITG turbulence. We conclude that discrete particle noise can also be a factor in the longest PIC simulations of Cyclone base case ITG turbulence. This does not affect the results reported in Ref. 12 because the simulations described in that paper used a very large number of particles and terminated before discrete particle effects became important. However, it does affect related simulations, which have been reported at major meetings. ${ }^{21}$

Fortunately, there is a literature on discrete particle noise. ${ }^{4,5,22-25}$ This literature allows us to quantify the level of discrete particle noise in any particular PIC simulation run, thereby casting the problem of discrete particle noise in PIC simulations as a code verification issue. ${ }^{26}$ A major result of this work is the diagnostics, described in Sec. III, which can be conveniently implemented in PIC simulations codes to aid in retrospectively monitoring the discrete particle noise in each simulation run. 


\section{Simulations of Cyclone base-case-like ETG turbulence}

The Cyclone base case plasma parameters, which were originally introduced as a benchmark for simulations of ITG turbulence, ${ }^{12}$ are $R_{0} / L_{T}=6.9, R_{0} / L_{n}=2.2, T_{e} / T_{i}=1.0$, $q=1.4$, and $s=(r / q) d q / d r=0.79$. These parameters have since been adopted in several numerical studies of ETG turbulence. ${ }^{6-11}$ We revisit these plasma parameters in this study of Cyclone base-case-like ETG turbulence in an effort resolve the differences between Refs. 6-8 and Refs. 9-11.

The plasma parameters used for the Cyclone base-case-like ETG turbulence simulations reported in Refs. 6-8 differs from those considered in Refs. 9-11 in that Refs. 6-8 did not include magnetic trapping (magnetic trapping is a finite-aspect-ratio effect which can be eliminated by setting $r / R_{0}=0$ ), while Refs. 9-11 included magnetic trapping (by taking $r / R_{0} \approx 0.18$ ). The inclusion of magnetic trapping makes only a small difference in the linear growth spectrum of ETG modes (see Fig. 1). However, this finite aspectratio effect appears to be the only difference between the plasma parameters used in the simulations reported in Refs. 6-8 and those used in Refs. 9-11. We investigate the effect of magnetically trapped electrons on Cyclone base-case-like ETG turbulence by comparing two sets of PG3EQ simulations, with $\left(r / R_{0}=0.18\right)$ and without $\left(r / R_{0}=0\right)$ magnetically trapped electrons. 


\section{II.A Cyclone base-case-like ETG turbulence without magnetic trapping}

We performed convergence studies in both particle number and flux-tube crosssection for Cyclone base-case-like ETG turbulence without magnetic trapping $\left(r / R_{0}=0\right)$ using the flux-tube PIC code, PG3EQ. ${ }^{13}$ Figure 2 shows $\chi_{e}(t) \equiv-\left\langle Q_{e}(t)\right\rangle / \nabla T_{0}$ from this convergence study, where $\nabla T_{0}$ is the equilibrium electron temperature gradient, $Q_{e}(t)$ is the radial electron heat flux, and the average is taken over the entire simulation volume. The particle number scan included runs at 2 particles/grid cell (blue curve in Fig. 2), 4 particles/grid cell (green curve in Fig. 2), 8 particles/grid cell (black curve in Fig. 2), and 16 particles/grid cell (red curve in Fig. 2). Convergence at late-times in flux-tube crosssection is demonstrated by comparing runs with a cross-section of $125 \rho_{e} \times 125 \rho_{e}$ $\left(128 \times 128\right.$ grid cells with a grid spacing $\left.\Delta x=\Delta y=0.9817 \rho_{e}\right)$ - the blue, green, and black curves in Fig. 2 - to a run with a cross-section of $250 \rho_{e} \times 62.5 \rho_{e}(256 \times 64$ grid cells with the same grid spacing) — the red curve in Fig. 2.

In each simulation run there is a burst of ETG turbulence during which the instantaneous electron heat transport approaches, and often exceeds the transport level reported in Refs. 6-8, $\chi \chi_{e} \approx 13\left(\rho_{e} / L_{T}\right) \rho_{e} v_{t e}$. Both the length and the intensity of this burst increases with the number of particles per grid cell. However, at late times the turbulent heat transport in all of these simulations drops to $\chi_{e} \approx 1.5\left(\rho_{e} / L_{T}\right) \rho_{e} v_{t e}$, even lower than the late-time electron heat transport reported in Refs. 9 and 10, $\chi \chi_{e} \approx 3.2\left(\rho_{e} / L_{T}\right) \rho_{e} v_{t e}$. The latetime electron heat transport in all of the PG3EQ simulations in our particle-number and 
box-size convergence study is nearly constant in time with $\chi_{e} \approx 1.5\left(\rho_{e} / L_{T}\right) \rho_{e} v_{t e}$. Hence, the late-time (but not the intermediate time) $\chi_{e} \approx 1.5\left(\rho_{e} / L_{T}\right) \rho_{e} v_{t e}$ from this study is converged in both particle number and box size.

In addition to exhibiting low heat transport at late times, these PG3EQ simulations exhibit the downshift in perpendicular wave-number observed in Refs. 9-11. Figure 3 shows the ETG fluctuation spectrum averaged over the radial variable $(x)$ and plotted vs. $k_{y} \rho_{e}$ from a PG3EQ simulation with a cross-section of $125 \rho_{e} \times 125 \rho_{e}$ and 8 particles/grid cell (corresponding to the black curve in Fig. 2). Comparing the ETG fluctuation spectrum in the late linear phase, $t=420 L_{T} / v_{t e}$ (red curve in Fig. 3 ) to the fluctuation spectrum well into the nonlinear phase, about 20 linear growth times later at $t=995 L_{T} / v_{t e}$ (black curve in Fig. 3) we see a down-shift in the perpendicular wave number from $k_{y} \rho_{e} \approx 0.35$ in the linear phase to $k_{y} \rho_{e} \approx 0.15$ in the nonlinear phase. This behavior is quite similar to that observed in Refs. 9-11 (c.f., Fig. 1 of Ref. 11), despite the much smaller dimension of our flux-tube in the bi-normal direction $\left(125 \rho_{e}\right)$ and correspondingly large spacing $\left(\Delta k_{y}=0.05 / \rho_{e}\right)$ between Fourier modes in $k_{y}$ (the bi-normal lies within the flux surface, perpendicular to $\boldsymbol{B}$ ). 


\section{II.B Cyclone base-case-like ETG turbulence with magnetic trapping}

Concern about the radial box size relative to the radial correlation length of the ETG "streamers" led us to substantially increase the flux-tube cross-section in our PG3EQ simulations of Cyclone base-case-like ETG turbulence with magnetic trapping $\left(r / R_{0}=0.18\right)$. However, neither the increase in flux-tube cross-section nor the inclusion of magnetic trapping made a qualitative change in our simulation results. Figure 4 shows a convergence study in particle number and flux-tube cross-section of Cyclone base-caselike ETG turbulence simulations. The particle number scan included runs with 2 particles/grid cell (green curve in Fig. 4), 4 particles/grid cell (blue curve in Fig. 4), and 16 particles/grid cell (red curve in Fig. 4). In these runs the flux-tube cross-section is held fixed at $500 \rho_{e} \times 125 \rho_{e}$ (512 grid cells in the radial direction and 128 cells in the bi-normal direction). Convergence in flux-tube cross-section at late times is demonstrated by comparing the late-time values of $\chi_{e}(t)$ from this particle-number convergence study with $\chi_{e}(t)$ from a simulation with a flux tube cross-section of $250 \rho_{e} \times 62.5 \rho_{e}(256 \times 64$ grid cells $)$ and 16 particles/grid cell shown by the black curve in Fig. 4. In every case there is a burst of ETG turbulence accompanied by substantial electron heat transport. As before, the duration and intensity of the burst of ETG turbulence increases with the number of particles per grid cell. At late times both the fluctuation intensity and the heat transport drop to a low level. The late-time electron heat transport observed in Cyclone base-caselike ETG simulations with magnetic trapping is independent of the number of particles 
per grid cell and the flux tube cross-section. Quantitatively, we find $\chi_{e} \approx 3\left(\rho_{e} / L_{T}\right) \rho_{e} v_{t e}$, in excellent agreement with the heat transport reported in Refs. 9-10, $\chi_{e} \approx 3.2\left(\rho_{e} / L_{T}\right) \rho_{e} v_{t e}$.

In addition to reproducing the heat transport reported in Refs. 9-10, we reproduce the downshift in wave number of the nonlinear spectrum relative to the linear spectrum. Figure 5 shows the fluctuation spectrum averaged over the radial variable $(x)$ and plotted vs. $k_{y} \rho_{e}$ late in the linear phase, $t=400 L_{T} / v_{t e}$ (red curve in Fig. 5) and at a time $30 / \gamma_{0} \approx$ $828 L_{T} / v_{t e}$ after the initial saturation of the ETG turbulence. The PG3EQ simulation again reproduces the downshift in the perpendicular wave-number seen in the global simulations of Ref. 9-11 (c.f., Fig. 1 of Ref. 11) despite the much smaller dimension of our flux-tube in the bi-normal direction $\left(62.5 \rho_{e}\right)$ and correspondingly large spacing $\left(\Delta k_{y}=\right.$ $0.1 / \rho_{e}$ ) between Fourier modes in $k_{y}$.

\section{II.C Late-time ETG potential fluctuations}

An examination of Figs. 2 through 5 leads one to conclude that at late times our PG3EQ simulations appear to be converged in both particle number and systems size. It would seem to follow that steady-state Cyclone base-case-like ETG turbulence produces very little heat transport, in agreement with Refs. 9-10, and in contradiction to Refs. 6-8. However, an examination of the late-time potential fluctuations in our simulations of Cyclone base-case-like ETG turbulence indicates that this conclusion is unwarranted. 
Figure 6 shows a grey-tone rendering of the mid-plane potential fluctuations both during the burst of ETG turbulence and at late times during the "steady-state" period when particle number and flux-tube cross-section convergence were achieved, and the heat flux was very low. It is obvious from Fig. 6 that the late-time potential fluctuations have little in common with the characteristic, large-scale ETG "streamers" seen during the earlier burst of ETG turbulence when the electron heat transport is large. There are no large-scale potential structures at late times in our PG3EQ simulations of Cyclone base-case-like ETG turbulence. Instead, the grey-tone rendering in Fig. 6 of the late-time potential fluctuations looks rather like the image on the screen of a TV set whose antenna has been disconnected. Since the late-time potential looks like shot-noise, we investigate the hypothesis that the late-time behavior of our PG3EQ simulations, and (by inference) the GTC simulations described in Refs. 9-11 are dominated by discrete particle noise.

\section{Discrete Particle Noise}

There is an extensive literature to draw upon when quantifying discrete particle noise. We will present elsewhere ${ }^{25}$ a detailed calculation of the gyrokinetic particle noise spectrum based on the test-particle superposition principle, which includes the self-Debye shielding of computer particles by other simulation particles. This calculation extends earlier calculations, such as that in Ref. 24, to include numerical corrections arising from solving the field equations on a discrete grid. Here we carry out a very simple derivation 
based on completely uncorrelated particles. This calculation differs from the more exact calculation by, at most, a factor of 2 . It does this well because only one plasma species is treated with discrete particles, while the adiabatic response from the other species provides approximately half of the shielding (some additional shielding is provided by the polarization term in the gyrokinetic Poisson equation). The treatment below loosely follows Chapter 12 of Ref. 23.

Particle-in-cell simulations represent field quantities, like the electrostatic potential $\phi$, on a discrete grid, $\left\{\boldsymbol{x}_{j}\right\}$, while the computer particles exist in a continuous space, $\boldsymbol{x}$. We restrict our attention to field quantities defined on a discrete grid and adopt the Fourier transform conventions:

$$
\begin{aligned}
& \phi_{j}=\sum_{\mathbf{k}} \phi_{\mathbf{k}} \exp \left(i \mathbf{k} \cdot \mathbf{x}_{j}\right) \\
& \phi_{\mathbf{k}}=\frac{1}{N_{G}} \sum_{j} \exp \left(-i \mathbf{k} \cdot \mathbf{x}_{j}\right) \phi_{j}
\end{aligned}
$$

where $\phi_{j}$ is the potential at the grid point located at $x_{j}$. Because the potential is defined on a discrete grid, its Fourier representation, $\phi_{\mathrm{k}}$ is defined on $-\pi / \Delta x \leq k_{x} \leq \pi / \Delta x$ (and similarly for $k_{y}$ and $k_{z}$ ).

The Fourier-space representation of the gyrokinetic Poisson equation ${ }^{27}$ is:

$$
\left\{1+\left[1-\Gamma_{0}\left(k_{\perp}^{2} \rho_{e}^{2}\right)\right]\right\}_{T}^{e \phi_{\mathbf{k}}}=\frac{S_{G}(\mathbf{k})}{N_{p}} \sum_{\mathbf{p}} S\left(\mathbf{k}_{\mathbf{p}}\right) \sum_{i} w_{i} J_{o}\left(k_{\perp} \rho_{i}\right) \exp \left(-i \mathbf{k}_{\mathbf{p}} \cdot \mathbf{x}_{i}\right)
$$


where the first term on the left-hand-side (LHS) represents Debye shielding by the passive, adiabatic species (ions in the case of ETG turbulence), while the term in square brackets on the LHS represents the polarization of the kinetic electron species. The righthand-side (RHS) includes explicit spatial filtering applied to the electrostatic potential on the field grid, $S_{G}(\boldsymbol{k})$; the total number of simulation particles, $N_{p}=n_{p} V$ (where $n_{p}$ is the number density of simulation particles and $V$ is the volume of the simulation); the individual particle weights, $w_{i}$; and the Bessel function, $J_{0}\left(k_{\perp} \rho_{i}\right)$, where $\rho_{i}$ is the gyroradius of the $i^{\text {th }}$ electron. The RHS also includes a sum over "grid aliases", 22,23 a consequence of the fact that the computer particles and, hence, their density, exist in a continuous space. Structure in the computer-particle phase space at wave numbers $k_{p x}$ lying in the interval $-\pi / \Delta x+p_{x}(2 \pi / \Delta x) \leq k_{p x} \leq \pi / \Delta x+p_{x}(2 \pi / \Delta x)$ (and similarly for $k_{y}$ and $k_{z}$,) appear on the grid at the "alias" wave number $k_{x}=k_{p x}-p_{x}(2 \pi / \Delta x)$ where $p_{x}$ is an integer (and similarly for $k_{y}$ and $k_{z}$ ). The amplitude of the aliased signal is reduced by $S\left(\mathbf{k}_{\mathbf{p}}\right)$, where $S(\mathbf{k})$ is the Fourier transform of the weighting function used to interpolate the particle density from the continuous space of computer particle positions, $\boldsymbol{x}$, onto the discrete field grid, $\left\{\boldsymbol{x}_{\boldsymbol{j}}\right\}$; and $\mathbf{p}$ denotes an integer vector with components $\left(p_{x}, p_{y}, p_{z}\right)$.

The gyrokinetic Poisson equation is easily solved for the electrostatic potential:

$$
\frac{e \phi_{\mathbf{k}}}{T}=\frac{S_{G}(\mathbf{k})}{N_{p}\left[2-\Gamma_{0}\left(k_{\perp}^{2} \rho_{e}^{2}\right)\right]} \sum_{\mathbf{p}} S\left(\mathbf{k}_{\mathbf{p}}\right) \sum_{i} w_{i} J_{o}\left(k_{\perp} \rho_{i}\right) \exp \left(-i \mathbf{k}_{\mathbf{p}} \cdot \mathbf{x}_{i}\right)
$$

Squaring this expression, we obtain the spectral density, 


$$
\begin{aligned}
& \left\langle\left|\frac{e \phi_{\mathbf{k}}}{T}\right|^{2}\right\rangle_{N}=\frac{S_{G}^{2}(\mathbf{k})}{\left[2-\Gamma_{0}\left(k_{\perp}^{2} \rho_{e}^{2}\right)\right]^{2}}\left\{\frac{\Gamma_{0}\left(k_{\perp}^{2} \rho_{e}^{2}\right)\left\langle w^{2}\right\rangle}{N_{p}} \sum_{\mathbf{p}} S^{2}\left(\mathbf{k}_{\mathbf{p}}\right)\right. \\
& \left.\quad+\frac{1}{N_{p}^{2}}\left\langle\sum_{\mathbf{p}, \mathbf{p}^{\prime}} S\left(\mathbf{k}_{\mathbf{p}}\right) S\left(\mathbf{k}_{\mathbf{p}^{\prime}}\right) \sum_{i \neq i^{\prime}} w_{i} w_{i^{\prime}} J_{0}\left(k_{\perp} \rho_{i}\right) J_{0}\left(k_{\perp} \rho_{i^{\prime}}\right) \exp \left[-i\left(\mathbf{k}_{\mathbf{p}} \cdot \mathbf{x}_{i}-\mathbf{k}_{\mathbf{p}^{\prime}} \cdot \mathbf{x}_{i^{\prime}}\right)\right]\right\rangle\right\}
\end{aligned}
$$

where $i$ and $i$ ' are indices for the computer particles, $\mathbf{p}$ and $\mathbf{p}$ ' are the grid-aliasing vectors, and the average is to be taken over an ensemble of realizations of the computer particles. In what follows we make use of the identity, valid for nearest-grid-point particle weighting used in PG3EQ, that for all $\mathbf{k}$,

$$
\sum_{\mathbf{p}} S^{2}\left(\mathbf{k}_{\mathbf{p}}\right) \equiv 1 \quad \text { (for nearest grid-point weighting). }
$$

The second term in the bracket on the RHS of Eq. (3) represents correlations between computer particle positions and weights. This term describes the contribution of waves, instabilities, and turbulence to the spectral density, as well as the correlations between particles that are responsible for the self-Debye shielding by particles of the kinetic species. We can provide an upper-bound on the discrete particle noise by ignoring the correlations among the computer particle positions and weights. The second term on the RHS then vanishes, and we are left with the fully uncorrelated fluctuation spectrum,

$$
\left\langle\left|\frac{e \phi_{\mathbf{k}}}{T}\right|^{2}\right\rangle_{N}=\frac{\left\langle w^{2}\right\rangle S_{G}^{2}(\mathbf{k}) \Gamma_{0}\left(k_{\perp}^{2} \rho_{e}^{2}\right)}{N_{p}\left[2-\Gamma_{0}\left(k_{\perp}^{2} \rho_{e}^{2}\right)\right]^{2}} \underset{|\mathbf{k}| \rightarrow 0}{\longrightarrow} \frac{\left\langle w^{2}\right\rangle}{N_{p}}
$$


In the limit $|\boldsymbol{k}| \rightarrow 0$, the fully uncorrelated spectrum goes to the mean-squared particle weight, $\left\langle w^{2}\right\rangle$, divided by the number of simulation particles.

It is our experience that the fully uncorrelated spectrum overstates the discrete particle noise at low $k$ by up to a factor of 2 . A more complete derivation of discrete particle noise, which includes the effects of Debye shielding by the kinetic species, will be presented elsewhere. ${ }^{25}$ When the self-Debye shielding is included, our estimate of the spectrum of discrete particle noise is reduced to

$$
\left\langle\left|\frac{e \phi_{\mathbf{k}}}{T}\right|^{2}\right\rangle_{H}=\frac{\left\langle w^{2}\right\rangle S_{G}^{2}(\mathbf{k}) \Gamma_{0}\left(k_{\perp}^{2} \rho_{e}^{2}\right)}{N_{p}\left[2-\Gamma_{0}\left(k_{\perp}^{2} \rho_{e}^{2}\right)\right]\left[2-\left(1-S_{G}(\mathbf{k}) d_{\|}(\mathbf{k})\right) \Gamma_{0}\left(k_{\perp}^{2} \rho_{e}^{2}\right)\right]} \underset{|\mathbf{k}| \rightarrow 0}{\longrightarrow} \frac{\left\langle w^{2}\right\rangle}{2 N_{p}}
$$

where $d_{\|}(\boldsymbol{k})$ is related to k-space representation of the differencing operator used to obtain the parallel electric field from the potential. In the limit of large- $k$, the term $S_{G}(\boldsymbol{k}) d_{\|}(\boldsymbol{k})$ vanishes and these two estimates of the discrete particle noise spectrum are identical. The effect of including self-Debye shielding is to reduce the estimate of the discrete particle fluctuation level by a factor of 2 at small $k$. In the limit that filtering/numerical effects are neglected, Eq. (5) agrees with the calculations of Refs. 24 and 5, modified for the case of one species being adiabatic.

Examining Eqs. (4) and (5), we see that our estimates of the noise spectrum depend only on the interpolation scheme used to assign the particle density to the field 
grid [which determines $S(\boldsymbol{k})$ ], the numerical algorithms used in the field solve [which determine $S_{G}(\boldsymbol{k}), \Gamma_{0}\left(k_{\perp}{ }^{2} \rho_{t h}{ }^{2}\right)$, and $\left.d_{\|}(\boldsymbol{k})\right]$, and the mean-squared particle weight, $\left\langle w^{2}\right\rangle$. The mean-squared particle weight has the interesting property that it is monotonically increasing in time (neglecting a small correction due to changes in the field energy). This follows from the entropy theorem of Lee and Tang, ${ }^{28}$ which relates the rate of increase in $\left\langle w^{2}\right\rangle$ to the heat flux:

$$
\frac{d\left\langle w^{2}\right\rangle}{d t} \approx \frac{2 \chi_{e}(t)}{L_{T}^{2}}
$$

This relation follows from the fact that $\delta f=f-f_{0} \approx-\left(x-x_{0}\right) d f_{0} / d x$. That is, the further a simulation particle diffuses from its initial position, the larger its weight must be to represent the difference between the current value of the distribution function and the local value of $f_{0}$.

Particle-in-cell simulation codes compute and save $\left\langle w^{2}\right\rangle$ as a function of time as the simulation progresses. In the event that $\left\langle w^{2}\right\rangle$ is not available, a reasonable approximation can be obtained by simply integrating the observed heat flux. Hence, all the information required to evaluate either of our estimates of the discrete particle noise spectrum is routinely available.

Typically, only a limited amount of fluctuation data is retained in simulations of plasma microturbulence. In the simulations described in Sec. II above we only retained 
the time history of the potential at the outboard mid-plane (labeled $z=0$ in PG3EQ's coordinate system). This allows us to compute the fluctuation spectrum at $z=0$, which must be compared with a mixed (Fourier and configuration-space) representation of the discrete particle noise spectrum,

$$
\begin{aligned}
\left\langle\left|\frac{e \phi_{k_{x}, k_{y}}(z)}{T}\right|^{2}\right\rangle & =\sum_{k_{z}}\left\langle\left|\frac{e \phi_{k_{x}, k_{y}, k_{z}}}{T}\right|^{2}\right\rangle \\
& \approx \frac{\left\langle w^{2}\right\rangle}{n_{p}\left(L_{x} L_{y} \Delta z\right)}\left\{\frac{\Delta z}{2 \pi} \int_{-\pi / \Delta z}^{\pi / \Delta z} \frac{S_{G}^{2} \Gamma_{0}}{\left[2-\Gamma_{0}\right]\left[2-\left(1-S_{G} d_{\|}\right) \Gamma_{0}\right]} d k_{z}\right\}
\end{aligned}
$$

In Figure 7 we compare the predicted noise spectrum plotted vs. $k_{y} \rho_{e}$ to the latetime fluctuation spectrum from the $250 \rho_{e} \times 62.5 \rho_{e}(256 \times 64$ grid cells) PG3EQ simulation of Cyclone base-case-like ETG turbulence with magnetic trapping $\left(r / R_{0}=0.18\right)$ shown in Figs. 4, 5, and 6. The spectrum has been averaged over both the radial coordinate and time $\left(2500 L_{T} / v_{t e}<t<3000 L_{T} / v_{t e}\right)$. Also shown is the fluctuation spectrum averaged over the time interval $1200 L_{T} / v_{t e}<t<1300 L_{T} / v_{t e}$ (that is, about 30 linear growth times after the initial saturation) together with the self-Debye shielded noise spectrum at that time. In Figure 8 we compare the predicted noise spectrum plotted vs. $k_{x} \rho_{e}$ to the latetime fluctuation spectrum from the same data set averaged over the bi-normal coordinate (y). The spectra shown in Figs. 7 and 8 differ largely because the field-solve in PG3EQ is asymmetric. Fourier techniques are use in the bi-normal $(y)$ direction, while finite difference techniques are used in the radial $(x)$ direction. In both Figs. 7 and 8 there is good agreement between the observed late-time fluctuation spectrum and the fullyuncorrelated noise spectrum; while there is excellent agreement with the self-Debye 
shielded noise spectrum. The only significant feature seen in the late-time signal and not in our prediction of the discrete particle noise spectrum is the longest wavelength zonal flow $\left(k_{x} \rho_{e}=0.025, k_{y} \rho_{e}=0\right)$, which does not contribute to radial transport. This is in contrast to the spectrum at earlier times $\left(1200 L_{T} / v_{t e}<t<1300 L_{T} / v_{t e}\right)$ during the burst of ETG turbulence, where $\chi_{e}$ still takes on substantial values $\left[\chi_{e} \approx 30-35\left(\rho_{e} / L_{T}\right) \rho_{e} v_{t e}\right]$ and the fluctuation spectrum at $k_{y} \rho_{e}=0.1$ is two orders of magnitude above the noise level (compare the green and chartreuse curves in Fig. 7). We conclude from this that the PG3EQ Cyclone base-case-like ETG turbulence simulations described in Sec. II are dominated by discrete particle noise at late times; and that the estimates of the discrete particle noise given above in Eqs. (4) and (5) accurately reproduce the fluctuation spectrum observed in PIC simulations dominated by discrete particle noise.

Comparisons between the observed fluctuation spectrum and the predicted noise spectrum provide a means of determining when a particular simulation run is dominated by discrete particle noise. However, this diagnostic is computationally intensive and requires the retention of substantial amounts of simulation data. A less computationally intensive diagnostic involves comparing the observed fluctuation intensity, $\left\langle/ e \phi /\left.T\right|^{2}\right\rangle$, with the fluctuation intensity expected from discrete particle noise alone,

$$
\left\langle\left|\frac{e \phi}{T}\right|^{2}\right\rangle=\sum_{\mathbf{k}}\left\langle\left|\frac{e \phi_{\mathbf{k}}}{T}\right|^{2}\right\rangle=\frac{\left\langle w^{2}\right\rangle}{n_{p} V_{\text {shield }}}
$$


where $n_{p}$ is the number density of computer particles, and the fully uncorrelated shielding volume is given by

$$
V_{\text {shield }}^{(N)} \equiv\left\{\frac{1}{(2 \pi)^{3}} \int d^{3} \mathbf{k} \frac{S_{G}^{2} \Gamma_{0}\left(k_{\perp}^{2} \rho_{e}^{2}\right)}{\left[2-\Gamma_{0}\left(k_{\perp}^{2} \rho_{e}^{2}\right)\right]^{2}}\right\}^{-1}
$$

while the self-Debye shielded volume is given by

$$
V_{\text {shield }}^{(H)} \equiv\left\{\frac{1}{(2 \pi)^{3}} \int d^{3} \mathbf{k} \frac{S_{G}^{2} \Gamma_{0}\left(k_{\perp}^{2} \rho_{e}^{2}\right)}{\left[2-\Gamma_{0}\right]\left[2-\left(1-S_{G} d_{\|}\right) \Gamma_{0}\right]}\right\}^{-1} .
$$

Alternatively, one can construct the fluctuation energy density,

$$
\frac{1}{2} m_{e} n_{e}\left\langle V_{E \times B}^{2}\right\rangle=-\frac{\omega_{p}^{2}}{\Omega_{c}^{2}}\left\langle\frac{\phi \nabla_{\perp}^{2} \phi}{8 \pi}\right\rangle .
$$

This has direct physical significance (the kinetic energy associated with the $E \times B$ motion), and is closely related to the transport coefficient, $D \approx\left\langle V_{E \nless B}{ }^{2}\right\rangle \tau_{c o r r}$. The contribution of the discrete particle noise to the fluctuation energy density is

$$
-\frac{\omega_{p}^{2}}{\Omega_{c}^{2}}\left\langle\frac{\phi \nabla_{\perp}^{2} \phi}{4 \pi}\right\rangle=n T \frac{\left\langle w^{2}\right\rangle}{n_{p} V_{\text {shield }}}\left\langle K_{\perp}^{2} \rho^{2}\right\rangle_{\text {noise }}
$$


where expressions for $V_{\text {shield }}$ are given above. The average over the discrete particle noise spectrum of the perpendicular wave vector is given by

$$
\left\langle K_{\perp}^{2} \rho^{2}\right\rangle_{\text {noise }}^{(N)} \equiv\left\{\frac{V_{\text {shield }}}{(2 \pi)^{3}} \int d^{3} \mathbf{k} \frac{K_{\perp}^{2}(\mathbf{k}) \rho^{2} S_{G}^{2}(\mathbf{k}) \Gamma_{0}\left(k_{\perp}^{2} \rho_{e}^{2}\right)}{\left[2-\Gamma_{0}\left(k_{\perp}^{2} \rho_{e}^{2}\right)\right]^{2}}\right\}
$$

for the fully uncorrelated noise spectrum, and by

$$
\left\langle K_{\perp}^{2} \rho^{2}\right\rangle_{\text {noise }}^{(H)} \equiv\left\{\frac{V_{\text {shield }}}{(2 \pi)^{3}} \int d^{3} \mathbf{k} \frac{K_{\perp}^{2}(\mathbf{k}) \rho^{2} S_{G}^{2}(k) \Gamma_{0}\left(k_{\perp}^{2} \rho_{e}^{2}\right)}{\left[2-\Gamma_{0}\right]\left[2-\left(1-S_{G} d_{\|}\right) \Gamma_{0}\right]}\right\}
$$

for the self-Debye shielded noise spectrum.

In figure 9 we compare our estimates of the fluctuation energy to that observed at the outboard mid-plane in the $250 \rho_{e} \times 62.5 \rho_{e}(256 \times 64$ grid cells) PG3EQ simulation of Cyclone base-case-like ETG turbulence with magnetic trapping $\left(r / R_{0}=0.18\right)$ shown in Figs. 4, 5, 6, 7, and 8. This diagnostic make it clear that (1) this simulation is dominated by discrete particle noise for $t>1000 L_{T} / v_{t e}$ in the sense that more than half of the fluctuation energy can be accounted for by discrete particle noise; and (2) essentially all 
of the fluctuation energy can be accounted for by discrete particle noise for $t>2000$ $L_{T} / v_{t e}$.

Figure 9 illustrates that PIC simulation of Cyclone base-case-like ETG turbulence is a pathological case in the sense that the ETG turbulences dies away at late-times, leaving only the discrete particle noise. This makes it difficult to develop a rule-ofthumb to use when evaluating the discrete particle noise diagnostics developed in this section. Fortunately, we have other examples of discrete particle noise in PIC simulations. In Fig. 10 we compare the observed fluctuation energy from PG3EQ simulations of Cyclone base-case ITG turbulence to the noise estimates. The drop in $\chi_{i}$ at late times $\left(t>500 \mathrm{a} / \mathrm{c}_{s}\right)$ occurs as the energy associated with the discrete particle fluctuations exceeds half of the total fluctuation energy. This suggests that, as a rule-ofthumb, we consider a PIC simulation to be dominated by discrete particle noise when more than $1 / 2$ of the total fluctuation energy can be attributed to discrete particle noise.

\section{Can discrete particle noise suppress ETG turbulence?}

We demonstrated in Sec. III that our PG3EQ simulations of Cyclone base-caselike ETG turbulence are dominated by discrete particle noise at late-times. However, this does not occur because the discrete particle noise rises to engulf the ETG signal. Instead, the ETG turbulence dies away, leaving behind the discrete particle noise (see Figs. 7 and 9). This raises the question of why the ETG turbulence died off. How can the small level 
of transport due to noise $\left[\chi_{\text {noise }} \approx 3\left(\rho / L_{T}\right) \rho v_{t}\right]$ suppress a much larger transport $[\chi \approx$ $\left.20\left(\rho / L_{T}\right) \rho v_{t}\right]$ due to the turbulence? Although $\chi_{\text {noise }}$ is small compared to the turbulent $\chi$ at earlier times, simple estimates of the damping rate due to this noise, $k_{y}{ }^{2} D_{\text {noise }}$, [with $D_{\text {noise }}$ $=(2 / 3) \chi_{\text {noise }}$ to account for the difference between the Braginskii definition of thermal conductivity and random walk diffusion coefficients] indicate that $\chi_{\text {noise }} \sim 3$, as seen at late times in Figs. 2 and 4, is of the right magnitude to stabilize all of the ETG modes in the simulation (see Fig. 1). The noise spectrum is nearly isotropic in the plane perpendicular to $\boldsymbol{B}$, so the noise causes comparable transport in both the radial and bi-normal directions; while the ETG turbulence is highly anisotropic in the plane perpendicular to $\boldsymbol{B}$ so that the turbulence mainly drives transport in the radial direction, while its shorter wave-length in the bi-normal direction leaves it vulnerable to noise-induced transport in this direction.

If the ETG turbulence is stabilized at a fixed value of $D_{\text {noise }}$ (and corresponding $\left.\chi_{\text {noise }}\right)$ we can expect PIC simulations of ETG turbulence act as a self-regulating system when the number of simulation particles is varied. The energy density [see Eqs. (8) and (12)] and transport ${ }^{25}$ associated with discrete particle noise increase with increasing $\left\langle w^{2}\right\rangle / n_{p}$ so one might expect the noise level to drop as the number density of simulation particles, $\mathrm{n}_{p}$, is increased. However, the noise-level increases in time as $\left\langle w^{2}\right\rangle / n_{p} \propto \int^{t} d t^{\prime}$ $\chi\left(t^{\prime}\right) / n_{p}$ [using the Lee-Tang entropy balance ${ }^{28}$ in Eq. (6) to express how the weights in a $\delta f$ algorithm grow in time]. As the number density of simulation particles $n_{p}$ is increased the simulations stay in a turbulent state with high $\chi$ for a longer period of time [see Figs. (2) and (4)] until the mean-squared particle weight, $\left\langle w^{2}\right\rangle$, builds to a level sufficient to offset the increase in $n_{p}$, and produce the same final $D_{\text {noise }}$ (and $\chi_{\text {noise }}$ ) independent of the 
number of simulation particles - exactly the behavior observed in the particle-number convergence studies of Sec. II.

\section{IV.A The Noise Test}

We further investigate the hypothesis that discrete particle noise can suppress ETG turbulence using the noise test of Bolton ${ }^{29}$ and $\operatorname{Lin}^{30}$ We implement the noise test by selecting a reference PG3EQ simulation of Cyclone base-case-like ETG turbulence from the flux-tube cross-section convergence study with magnetic trapping $\left(r / R_{0}=0.18\right)$ illustrated in Fig. 4. We consider the case with a flux-tube cross-section $250 \rho_{\mathrm{e}} \times 62.5 \rho_{\mathrm{e}}$ (256×64 grid cells). Like the other simulations illustrated in Fig. 4, the late-time behavior of this simulation was dominated by discrete particle noise. When this simulation ended at $t=3000 L_{T} / v_{t e}$ the mean-squared particle weight was $\left\langle w^{2}\right\rangle_{\text {fina }} \approx 7.85 \times 10^{4}\left(\rho_{e} / L_{T}\right)^{2}$. If our hypothesis is correct, this mean-squared particle weight should, by itself, be sufficient to suppress Cyclone base-case-like ETG turbulence. The "noise test" investigates this hypothesis by re-initializing the simulation with all parameters set as they were at the beginning of the reference simulation except for the mean-squared particle weight. The initial weights of individual particles are then chosen from a random distribution scaled such that the mean-square weight, $\left(w^{2}\right\rangle_{\text {initial }}$, is proportional to the final mean-squared particle weight from our reference simulation, $\left\langle w^{2}\right\rangle_{\text {final }}$. In electrostatic $\delta$ PIC simulations, any "memory" of past events is encoded into the particle phase variables, so this test effectively erases all memory of the previous 
burst of ETG turbulence other than the discrete particle noise level as quantified by the mean-squared particle weight.

Figure 11 shows the result when we re-initialized this simulation with $\left\langle w^{2}\right\rangle_{\text {initial }}=$ $\left\langle w^{2}\right\rangle_{\text {final }}$ (red curve), $\left\langle w^{2}\right\rangle_{\text {initial }}=(1 / 2)\left\langle w^{2}\right\rangle_{\text {final }}$ (blue curve), $\left\langle w^{2}\right\rangle_{\text {initial }}=(1 / 4)\left\langle w^{2}\right\rangle_{\text {final }}$ (gold curve), $\left\langle w^{2}\right\rangle_{\text {initial }}=(1 / 8)\left\langle w^{2}\right\rangle_{\text {final }}$ (green curve), $\left\langle w^{2}\right\rangle_{\text {initial }}=(1 / 32)\left\langle w^{2}\right\rangle_{\text {final }}$ (chartreuse curve). When the simulation is restarted with $\left\langle w^{2}\right\rangle_{\text {initial }}=\left\langle w^{2}\right\rangle_{\text {final }}$ (red curve in Fig. 11), $\chi_{e}$ rises to the value it had at the end of the reference run $\left[\chi_{e} \approx 3\left(\rho_{e} / L_{T}\right) \rho_{e} v_{t e}\right]$ over a time interval of $2.5 L_{T} / v_{t e}$ (this initial transient is too short to be resolved in Fig. 11), and stays at approximately this value over the remainder of the simulation (a time interval of about $\left.1000 L_{T} / v_{t e}\right)$. While some low-level potential fluctuations with real frequencies satisfying the linear dispersion relation of the ETG modes are present after the restart in this run, it shows no evidence of instability, remaining at fluctuation intensities consistent with our estimate of the self-shielded noise spectrum.

The behavior of the restart with $\left\langle w^{2}\right\rangle_{\text {initial }}=(1 / 2)\left\langle\left(w^{2}\right\rangle_{\text {final }}\right.$ (blue curve in Fig. 11) is similar. The electron heat flux, measured by $\chi_{e}$, initially rises to a somewhat lower value $\left[\chi_{e} \approx 2\left(\rho_{e} / L_{T}\right) \rho_{e} v_{t e}\right]$ over the initial time interval of $2.5 L_{T} / v_{t e}$, and then increases very slowly to $\chi_{e} \approx 3\left(\rho_{e} / L_{T}\right) \rho_{e} v_{t e}$ over the remainder of the simulation. When the restart is initialized with progressively lower mean-squared particle weights the electron heat transport initially rises to progressively lower values: for $\left\langle w^{2}\right\rangle_{\text {initial }}=(1 / 4)\left\langle w^{2}\right\rangle_{\text {final }}$ (gold curve in Fig. 11) $\chi_{e}$ rises to about $1.2\left(\rho_{e} / L_{T}\right) \rho_{e} v_{t e}$ over the initial time interval; for 
$\left\langle w^{2}\right\rangle_{\text {initial }}=(1 / 8)\left\langle w^{2}\right\rangle_{\text {final }}$ (green curve in Fig. 11) $\chi_{e}$ rises to about $0.7\left(\rho_{e} / L_{T}\right) \rho_{e} v_{t e}$ over the initial time interval; and for $\left\langle w^{2}\right\rangle_{\text {initial }}=(1 / 32)\left\langle w^{2}\right\rangle_{\text {final }}$ (chartreuse curve in Fig. 11) $\chi_{e}$ rises to about $0.2\left(\rho_{e} / L_{T}\right) \rho_{e} v_{t e}$ over the initial time interval. These small initial values of $\chi_{e}$ are more easily seen in the expanded scale of Fig. 16. In each restart with $\left\langle w^{2}\right\rangle_{\text {initial }}<\left\langle w^{2}\right\rangle_{\text {final }}$, we observe linear growth of ETG modes accompanied by an increase in $\chi_{e}$ from these lower initial values. The maximum in $\chi_{e}$ during the initial burst of ETG turbulence increases with decreasing $\left(w^{2}\right)_{\text {initial }}$.

The measured linear growth rates of ETG modes also increase with decreasing $\left\langle w^{2}\right\rangle_{\text {initial }}$. The linear growth of ETG modes after the restart is examined in Fig. 12. In non-linear simulations like those described here we are only able to measure the linear growth rate of the dominant Fourier mode in each restart. The dominant mode in the initial simulation (see black curve in Fig. 12) is $k_{y} \rho_{e}=0.3$, with the measured growth rate of $\gamma=0.0381 \pm 0.0005 v_{t e} / L_{T}$ in reasonable agreement with the linear growth rate of 0.037 obtained from GS2. ${ }^{7,31}$ The dominant mode in the restart with $\left\langle w^{2}\right\rangle_{\text {initial }}=\left\langle w^{2}\right\rangle_{\text {final }}$ (red curve) is $k_{y} \rho_{e}=0.1$ (the longest wavelength mode in this simulation). A least-squares fit to the slope of this curve over the time-interval after the restart of $100 L_{T} / v_{t e}<t<400$ $L_{T} / v_{t e}$ is consistent with no linear growth, $\gamma=0.00045 \pm 0.00072 v_{t e} / L_{T}$. The dominant mode in the restart with $\left\langle w^{2}\right\rangle_{\text {initial }}=(1 / 2)\left\langle w^{2}\right\rangle_{\text {final }}$ (blue curve) is again $k_{y} \rho_{e}=0.1$. A leastsquares fit to the slope of this curve (in the semi-log representation shown) over the timeinterval after the restart of $100 L_{T} / v_{t e}<t<1000 L_{T} / v_{t e}$ yields a weakly growing mode with $\gamma=0.00052 \pm 0.00022 v_{t e} / L_{T}$. The error bars represents the change in the slope of the 
linear fit required to double the mean-squared error. The dominant mode in the restarts with $\left\langle w^{2}\right\rangle_{\text {initial }}=(1 / 4)\left\langle w^{2}\right\rangle_{\text {final }}$ (gold curve), $\left\langle w^{2}\right\rangle_{\text {initial }}=(1 / 8)\left\langle w^{2}\right\rangle_{\text {final }}$ (green curve), and $\left\langle w^{2}\right\rangle_{\text {initial }}=(1 / 32)\left\langle w^{2}\right\rangle_{\text {final }}$ (chartreuse curve) is $k_{y} \rho_{e}=0.2$. The corresponding growth rates are $0.0042 \pm 0.0004 v_{t e} / L_{T}\left[\left\langle w^{2}\right\rangle_{\text {initial }}=(1 / 4)\right], 0.029 \pm 0.007 v_{t e} / L_{T}\left[\left\langle w^{2}\right\rangle_{\text {initial }}=(1 / 8)\right]$, and $0.0168 \pm 0.008 v_{t e} / L_{T}\left[\left(w^{2}\right)_{\text {initial }}=(1 / 32)\right]$.

We conclude from this noise test that discrete particle noise suppresses both ETG turbulence and the associated anomalous electron heat transport by suppressing the linear growth of ETG modes. The critical value of the mean-squared particle weight required to suppress bursts of heat transport for Cyclone base-case-like ETG turbulence, $\left\langle w^{2}\right\rangle_{c r i t}$ lies within the interval $0.25\left\langle w^{2}\right\rangle_{\text {final }}<\left\langle w^{2}\right\rangle_{\text {crit }}<0.5\left\langle w^{2}\right\rangle_{\text {final }}$ (see Fig. 11); while the critical value of the mean-squared particle weight required to suppress linear growth of ETG modes lies within the interval $0.5\left\langle w^{2}\right\rangle_{\text {final }}<\left\langle w^{2}\right\rangle_{\text {crit }}<\left\langle w^{2}\right\rangle_{\text {final }}$ (see Fig. 12). 


\section{How discrete particle noise suppresses ETG turbulence}

Both the PG3EQ simulations described in Sec. II and the GTC simulations described in Ref. 9-11 treat the $E_{\|}$term in the "partially linearized" approximation. In this approximation both the magnetic moment and the kinetic energy of the simulation particles are conserved. Only the particle positions and weights evolve in time. It follows that the $90^{\circ}$ scattering time of the computer particles is infinite in the presence of discrete particle noise. Discrete particle noise does affect the particle positions through their $E \times B$ drift in the potential fluctuations computed in Sec. III. Hence, the leadingorder self-consistent "collisional" effect in partially-linearized PIC simulations is the spatial diffusion due to the discrete particle noise.

\section{V.A Linear Stabilization of ETG Modes by Diffusion}

Just as collisional scattering in velocity space must be included when computing the linear dispersion relation of experimental plasmas, we must include the spatial diffusion due to discrete particle noise when computing the linear dispersion relation of our computer plasma. The ETG turbulence simulations described in Sec. II and Ref. 9-11 treat the ions as a passive species entering the problem only through the Debye shielding 
term in the field equation. This term does not involve any time derivatives, so all the frequency dependence in the computer dispersion relation arise from the kinetic electron species. We model the effects of diffusion due to discrete particle noise by including a diffusion operator, $D_{\text {noise }} \nabla_{\perp}^{2} h$, in the electron kinetic equation, where $D_{\text {noise }}$ is the testparticle diffusion rate of the computer particles due to their interaction with the spectrum of discrete particle noise and $h$ is the non-adiabatic part of the electron distribution function. By applying diffusion only to the non-adiabatic part of the distribution function we preserve the property that there is no damping of a Boltzmann distribution, which is already in thermodynamic equilibrium. ${ }^{32,33}$ Future work could go beyond this test-particle diffusion approach by calculating an improved scattering operator for noise that would include back-reaction effects, to insure that the net particle flux is zero. (ETG/ITG turbulence when one species is adiabatic leads to a net heat flux but no net particle flux.)

The fastest growing linear mode at each value of $k_{y}$ from this model dispersion relation is found using the GS2 code. ${ }^{7,31}$ The variation of the real frequency and growth rate of CYCLONE base-case-like ETG turbulence with $D_{\text {noise }}$ is shown in Fig. 13 . The real part of the mode frequency is barely affected by $D_{\text {noise, }}$ while the growth rates decrease nearly linearly with increasing $D_{\text {noise }}$ at each value of $k_{y}$.

$$
\chi\left(k_{y}, D_{\text {noise }}\right) \approx \gamma\left(k_{y}, D_{\text {noise }}=0\right)-\alpha_{\text {noise }} k_{y}^{2} D_{\text {noise }} .
$$

A least-squares fit to the solutions of our model dispersion relation displayed in Fig. 13 yields $\alpha_{\text {noise }} \approx 2.4$. However, the random restart noise tests indicate that $\alpha_{\text {noise }} \approx 2.4$ 
overstates the stabilizing effect of discrete particle noise. Figure 14 shows that $\alpha_{\text {noise }} \approx 1$ gives a reasonable fit to the measured growth rates, as a function of $D_{\text {noise }}$.

We conclude that discrete particle noise suppresses ETG instabilities in PIC simulations by causing spatial diffusion of the simulation particles. For each Fourier mode when the discrete particle noise is large enough that $D_{\text {noise }} \approx \chi\left(k_{y}\right) / k_{y}{ }^{2}$, where $\chi\left(k_{y}\right)$ is the linear growth rate in the absence of discrete particle noise, the linear instability at that value of $k_{y}$ is suppressed.

\section{V.B Computing $D_{\text {noise }}$ and $\chi_{\text {noise }}$}

The spatial diffusion of computer particles caused by the spectrum of discrete particle noise derived in Sec. III above has been computed $^{25}$ using the shielded testparticle superposition principle with a resonance-broadening type of renormalization. The resulting test-particle diffusion due to the noise fluctuations is

$$
D_{\text {noise }}=\frac{1}{12}\left(\frac{c T}{e B}\right)^{2}\left\langle\left|\frac{e \phi}{T}\right|^{2}\right\rangle_{\text {noise }} \frac{V_{\text {Shield }}^{(H)} k_{\perp N}^{2}}{V_{S h, 2}} \frac{3.05}{k_{\| \max } v_{t e} \sqrt{2}} \log \left[1+\frac{k_{\| \max } v_{t e} \sqrt{2}}{3.05\left(D_{\text {noise }} k_{\perp N}^{2}+V_{*}\right)}\right] \text {, }
$$


where $\left\langle|e \phi / T|^{2}\right\rangle_{\text {noise }}$ and $V_{\text {shield }}^{(H)}$ are given by Eqs. (8) and (9). The perpendicular wave number (averaged over the noise spectrum and including some FLR effects), $k_{\perp N}$, is defined by

$$
k_{\perp N}^{2}=\frac{V_{S h, 2}}{V} \sum_{\mathbf{k}} \hat{k}_{\perp}^{2} \frac{\Gamma_{0}^{2}\left(k_{\perp}^{2} \rho_{t h}^{2}\right) S_{G}^{2}(\mathbf{k})\left(\sum_{\mathbf{p}} S^{2}\left(\mathbf{k}_{\mathbf{p}}\right)\right)^{2}}{\frac{1}{3}\left[2-\Gamma_{0}+\Gamma_{0} S_{G} d_{\|} \sum_{\mathbf{p}} S^{2}\right]\left[2-\Gamma_{0}+\frac{1}{2} \Gamma_{0} S_{G} d_{\|} \sum_{\mathbf{p}} S^{2}\right]},
$$

where $\hat{k}^{2}=\hat{k}_{x}^{2}+\hat{k}_{y}^{2}$, the Fourier transform of the discrete analog of $\partial / \partial x$ is denoted by $i \hat{k}_{x}=i k_{x} \operatorname{dif}\left(k_{x} \Delta x\right)\left(\right.$ and similarly for $\left.\hat{k}_{y}\right), \operatorname{dif}(x)=\sin (x) / x$, and

$$
\frac{1}{V_{S h, 2}}=\frac{1}{V} \sum_{\mathbf{k}} \frac{\Gamma_{0}^{2}\left(k_{\perp}^{2} \rho_{t h}^{2}\right) S_{G}^{2}(\mathbf{k})\left(\sum_{\mathbf{p}} S^{2}\left(\mathbf{k}_{\mathbf{p}}\right)\right)^{2}}{\frac{1}{3}\left[2-\Gamma_{0}+\Gamma_{0} S_{G} d_{\|} \sum_{\mathbf{p}} S^{2}\right]\left[2-\Gamma_{0}+\frac{1}{2} \Gamma_{0} S_{G} d_{\|} \sum_{\mathbf{p}} S^{2}\right]} .
$$

The cut-off in the parallel part of the filters is $k_{\| \max }$, defined as

$$
k_{\|, \max }=\frac{\pi}{L_{z}} \sum_{\mathbf{k}} S_{G \|}^{2}(\mathbf{k})\left(\sum_{\mathbf{p}} S_{\|}^{2}\left(\mathbf{k}_{\mathbf{p}}\right)\right)^{2} \approx \frac{1}{2} \int d k_{\|} S_{G \|}^{2}\left(k_{\|}\right)\left(\sum_{\mathbf{p}} S_{\|}^{2}\left(k_{\|}\right)\right)^{2} .
$$

The expression for $D_{\text {noise }}$ in Eq. (16) is of the form $D_{\text {noise }} \sim V_{E \times B}^{2} / v$, where $v$ is a decorrelation rate, and so is dimensionally correct and has the straightforward physical 
interpretation of particle diffusion due to random $\mathrm{E} \times \mathrm{B}$ motion. This calculation is "renormalized" in the sense that the decorrelation rate depends on $D_{\text {noise }}$ itself, and for this reason $D_{\text {noise }}$ appears inside the logarithm on the RHS of Eq. (16). In the strong noise limits, $D_{\text {noise }} k_{\perp N}^{2} \gg k_{\|, \max } v_{t}, v_{*}$, (which usually applies at late times in the noisedominated cases we show in this paper), the logarithmic factor in Eq. (16) can be expanded to give a familiar result that the decorrelation rate is of order $D_{\text {noise }} k_{\perp N}^{2}$.

The various approximations involved in the test-particle theory, and in the intermediate integrals used to obtain these results will be presented elsewhere. ${ }^{25}$ In the $\nu_{*}=0$ limit the derivation of Eq. (16) is based on a systematic theoretical calculation with no free parameters adjusted to match simulation data. The coefficient of 3.05 comes not from a fit to simulation data but, rather, from a fit to a numerical evaluation of the correlation time expressed as an integral involving the plasma dispersion function. This fit agrees with a numerical evaluation of the correlation time to within $\pm 12 \%$.

The $\nu_{*}$ term inside the logarithm in Eq. (16) provides qualitative estimates of decorrelation rates from mechanisms otherwise neglected in the noise theory of Ref. 25. We take $v_{*}=v_{\text {turb }}+v_{B, \text { shear }}+v_{\text {tor }}$, where $v_{\text {turb }}, v_{B, \text { shear }}$, and $v_{\text {tor }}$ are approximations for decorrelation rates due to turbulence, magnetic shear, and dispersion in the toroidal precession. The latter two mechanisms are modest corrections. They provide a lower bound on the total decorrelation rate, and have the effect of slightly reducing the estimate of $D_{\text {noise. }}$. The turbulent decorrelation disappears at late times when the turbulence is suppressed by noise, so the late-time calculations of $D_{\text {noise }}$ are independent of our 
expression for $v_{\text {turb }}$ and only weakly dependent on our assumptions about $v_{B, \text { shear }}$ and $v_{t o r}$ (see Fig. 15). Note that the theory for $D_{\text {noise }}$ and $\chi_{\text {noise }}$ agrees very well with the observed $\chi$ (compare Fig. 15 with Figs. 4 and 16) at late time, when the noise dominates.

The turbulent decorrelation rate is estimated by the rate at which noise fluctuations (primarily at high $k$ ) are sheared by an assumed spectrum of turbulent fluctuations at lower $k, v_{\text {turb }} \approx k_{\perp 0} k_{r 0} D_{\text {turb }}\left(k_{\perp N}^{2} / k_{\perp 0}^{2}\right)^{3 / 4}$, where $D_{\text {turb }}=\operatorname{Max}\left[(2 / 3) \chi_{o b s}-D_{\text {noise }}\right.$, 0], $\chi_{o b s}$ is the heat conductivity observed in a simulation, and $k_{\perp 0}^{2}=k_{y 0}^{2}+k_{r 0}^{2}$. For the purposes of this approximate model of turbulent decorrelation we choose $k_{y} \rho=0.15$ and $k_{r 0} \rho \approx 0.044$ as typical ETG turbulence wave numbers (note our results are insensitive to this approximate model at late times when the turbulence is suppressed). The magnetic shear de-correlation rate is approximated as $v_{B, s h a r}=k_{\perp N} \hat{s} v_{t e} /(q R)$, and the de-correlation due to particles having a spread in toroidal $\nabla B$ and curvature drift speeds is approximated as $v_{t o r}=k_{\perp N} v_{t e} /(\sqrt{2} R)$. For Cyclone base-case-like ETG turbulence we find $v_{B, \text { shear }} \approx 0.0475 v_{t e} / L_{T}$ and $v_{\text {tor }} \approx 0.060 v_{\text {te }} / L_{T}$.

The test-particle-based heat diffusivity $\chi_{\text {noise }}(t)=(3 / 2) D_{\text {noise }}(t)$ is shown in Fig. 16. We find excellent agreement between the theoretical predictions of the heat transport due to discrete particle noise and our simulation results as the mean-squared particle weight is varied by a factor of 32 in the noise tests described in Sec. IV. This correspondence between our estimate of $\chi_{\text {noise }}$ and the simulation results demonstrates the accuracy of our estimate of $D_{\text {noise }}$ [Eq. (16)] and supports our expressions for $v_{B, \text { shear }}$ and 
$v_{t o r}$ which are important to the determination of $D_{\text {noise }}$ at low mean-squared particle weight employed as part of this noise test. Figure 16 also demonstrates that essentially all of the electron heat transport is accounted for by discrete particle noise at late times in our PIC simulations of ETG turbulence.

Encouraged by the success of Eq. (16) in predicting $\chi_{\text {noise }}$ we extended the noise test of Sec. IV to include restarts in which the initial mean-squared particle weight, $\left\langle w^{2}\right\rangle_{\text {initial }}$, exceeded the mean-squared particle weight at the end of our reference simulation, $\left\langle w^{2}\right\rangle_{\text {final }}$. Figure 17(a) shows Cyclone base-case-like ETG simulations with $\left\langle w^{2}\right\rangle_{\text {initial }}=16\left\langle w^{2}\right\rangle_{\text {final }}$ (black curves) and $\left\langle w^{2}\right\rangle_{\text {initial }}=4\left\langle w^{2}\right\rangle_{\text {final }}$ (red curves), together with $\left\langle w^{2}\right\rangle_{\text {initial }}=\left\langle w^{2}\right\rangle_{\text {final }}$ (blue curves). In all of these additional cases the theory presented above provides a good estimate to the observed value of $\chi_{\text {noise }}$. Combining the noise test data from both Figs. 16 and 17(a), we have scanned $\left\langle w^{2}\right\rangle_{\text {initial }}$ over a dynamic range of 512. Figure 17(b) shows that our theoretical prediction provides a good fit to this simulation data over this entire range.

The simulations with $\left.\left\langle w^{2}\right\rangle_{\text {initial }}\right\rangle\left\langle w^{2}\right\rangle_{\text {final }}$ also allow us to address the suggestion made by some that the late-time state with $\chi_{e} \approx 3$ seen in Fig. 4 or in the results reported in Ref. 9 reflects the steady state ETG turbulence level in the absence of discrete particle noise, while the earlier high transport results from a transient overshoot. A change in initial conditions (like choice of a larger initial mean-squared particle weight) might temporarily drive larger fluctuations and higher transport. However, the simulation must eventually return to the $\chi_{e} \approx 3$ if that reflects the steady-state turbulence in the absence of 
discrete particle noise. If $\chi_{e} \approx 3$ results from the accumulation of discrete particle noise (as argued above), then choosing $\left\langle w^{2}\right\rangle_{\text {initial }}>\left\langle w^{2}\right\rangle_{\text {fnnal }}$ will simply further increase the discrete particle noise resulting in higher transport levels, consistent with Eq. (16), which persist indefinitely. Fig. 17(a) shows the restarts at higher $\left\langle w^{2}\right\rangle_{\text {initial }}$ have persistently higher transport, providing further evidence that the late time results from these runs are due to discrete particle noise. Evidence for this can also be seen in the particle number scan in Figs. 2 and 4, which show that the transition from the high transport phase to the $\chi_{e} \approx 3$ phase occurs at a later time as the number of particles increases. This correlation with particle number indicates a process affected by noise.

\section{V.C Estimates of $\gamma\left(k_{y}, D_{\text {noise }}(t)\right)$}

We may obtain an estimate of the net linear ETG growth rates as a function of time by combining our approximate expression for the linear growth rate in the presence of discrete particle noise, Eq. (15), with our expression for the noise diffusion as a function of mean-squared particle weight, Eqs. (8) and (16). Figure 18(a) shows the net linear ETG growth rates vs. time for the PG3EQ simulation at $r / R=0.18$ with flux-tube cross-section $250 \rho_{\mathrm{e}} \times 62.5 \rho_{\mathrm{e}}(256 \times 64$ grid cells), and 16 particles/cell (the reference simulation in the noise test of Sec. IV). In preparing Fig. 18 we took $\alpha_{\text {noise }}=1.0$, the best fit to the random weight restart tests of Fig. 14, corresponding to a somewhat weaker dependence of $\gamma$ on $D_{\text {noise }}$ than that obtained from the model dispersion relation of Sec. V.A. Despite adopting this weaker dependence of $\gamma$ on $D_{\text {noise }}$, we predict that all ETG 
modes present in this simulation are stabilized by discrete particle noise at late times $\left(t>1650 L_{T} / v_{t e}\right)$.

Figure 18(b) shows the intensity of the first five Fourier modes in the bi-normal (y) coordinate (averaged over the radial coordinate) versus time. If the saturation of these modes were solely due to the modification of their linear growth rate by discrete particle noise, then we would expect each Fourier mode to reach is maximum intensity when its net linear growth rate passes through zero. Comparing Figs. 18(a) and 18(b), we see that this is the case for the longest wavelength mode $\left(k_{y} \rho=0.1\right)$. Figure 18(a) indicates that the noise is large enough to stabilize this mode (and thus all modes), at late times ( $t>1650$ $L_{T}\left(v_{t}\right)$ in this simulation.

There is also evidence that the intermediate-time $\left(650 L_{T} / v_{t}<t<1000 L_{T} / v_{t}\right)$ saturation is strongly influenced by discrete particle noise. The intermediate-time transport and fluctuation levels are not converged in particle number (c.f., Figs. 2 and 4), while Fig. 18(a) indicates that the discrete particle noise is large enough to significantly modify the linear growth rates of the $k_{y} \rho_{e}=0.3$ and $k_{y} \rho_{e}=0.2$ modes over this interval.

It appears that that particle number convergence is beginning to be achieved for 16 particles/cell at early times $\left(t<650 L_{T} / v_{t e}\right)$ in these simulations. Comparing Figs. 18 and 19 , we see that the initial saturation of the $k_{y} \rho_{e}=0.3$ mode at $t=400 L_{T} / v_{t}$ occurs before the corresponding linear growth is strongly modified by discrete particle noise. 
However, Fig. 4 indicates that the heat transport is not yet fully converged in particle number over this time interval.

We conclude that modification of the linear growth rate due to discrete particle noise is responsible for the disappearance of the ETG turbulence at late times $(t>1650$ $L_{T} / v_{t e}$ ), and that this modification of the linear growth rates is affecting the saturation of the ETG turbulence even at intermediate times $\left(650 L_{T} / v_{t e}<t<1000 L_{T} / v_{t e}\right)$ in our PIC simulations of Cyclone base-case ETG turbulence with 16 particles /cell.

\section{Summary and Conclusions}

We quantitatively reproduced key results from GTC gyrokinetic particle-in-cell simulations of ETG turbulence using the flux-tube particle-in-cell code PG3EQ. Both our PG3EQ gyrokinetic particle-in-cell simulations and the GTC simulations of Cyclone base-case-like ETG turbulence begin with the linear growth of ETG modes, followed by a burst of ETG turbulence. Our simulations reproduced the evolution of the turbulent ETG spectrum observed in the GTC simulations from an initial peak near $k_{\perp} \rho_{e} \approx 0.3$ in the linear phase of the simulation to a peak at longer wavelength, $k_{\perp} \rho_{e} \approx 0.1$, late in the burst of ETG turbulence.

The burst of ETG turbulence dies away at late times and the electron heat transport drops to a low level, $\chi_{e} \approx 3\left(\rho_{e} / L_{T}\right) \rho_{e} v_{t e}$, in agreement with the late-time GTC 
result. ${ }^{9,10}$ However, we also found excellent agreement between the fluctuation spectrum observed at late-times in our PG3EQ simulations and the fluctuation spectrum produced by discrete particle noise. This leads us to conclude that both these PG3EQ simulations and, by inference, the GTC simulations we have reproduced are dominated by discrete particle noise at late times.

We demonstrated that the transport level at late times in our PG3EQ simulations depends only on the discrete particle noise as quantified by the mean-squared particle weight and the number of particles per smoothing volume. We evaluated the test-particle diffusion of computer particles caused by the discrete particle noise ${ }^{25}$ and demonstrated that the discrete particle noise observed at late times in these simulations produces enough diffusion to suppress linear growth of ETG modes. Because discrete particle noise is a numerical artifact of the Monte-Carlo-like PIC simulation method we conclude that the late-time behavior of both the PG3EQ simulations reported here and, by inference, the GTC simulations reported in Refs. 9-11 do not model ETG turbulence as it occurs in experimental plasmas.

At earlier times, during the burst of ETG turbulence, we find that both the intensity of the ETG turbulence and the magnitude of the electron heat transport increases with increasing numbers of simulation particles per grid cell. We have demonstrated that modifications to the linear growth rate due to the particle diffusion resulting from "collisions" between discrete particles contributes to the evolution of the ETG spectrum toward long wavelength. Since similar numbers of simulation particles/grid cell were 
employed both in our PG3EQ simulations and in GTC simulations we have reproduced (we used 3 to 6 times more particles per smoothing volume than we believe were used in the GTC simulations reported in Ref. 9), we conclude that neither our PG3EQ simulations nor these GTC simulations were well converged in particle number during the burst of ETG turbulence. Hence, both the PG3EQ simulations of Cyclone base-caselike ETG turbulence and the GTC simulations we have reproduced have little to say about the anomalous electron heat transport associated with ETG turbulence in experimental plasmas. However, we do note that the relatively high electron heat transport and the intermittent behavior of the ETG turbulence at early times in PG3EQ simulations with more than 2 particles/grid cell is reminiscent of the ETG turbulence seen in Refs. 6-8, providing qualitative confirmation of their results.

A theory of noise based on the test-particle superposition principle ${ }^{24,25}$ with no adjustable fitting parameters, agrees extremely well with the simulations at late times when the noise dominates, in both the spectrum of fluctuations and the transport driven by the noise. The predicted transport coefficient $\chi_{\text {noise }}$ agrees well with the simulations in random restart tests where the average squared weight (and thus the fluctuation energy) is scanned over a factor of 512. Although in this paper we have presented a logical sequence starting with the PG3EQ simulations first and comparing with the noise theory later, the theory (which will be presented in more detail elsewhere ${ }^{25}$ ) actually came first and predicted that PIC simulations would be dominated by noise at late times for this range of particles/cell. 
There are differences between the GTC and PG3EQ simulations that may change some details of how these codes are affected by discrete particle noise. However, these differences will not affect the overall conclusion that both the PG3EQ simulations reported here and the GTC simulations reported in Refs. 9-11 are dominated by discrete particle noise at late times. The noise in the PG3EQ simulations grew until it was large enough to stabilize all of the ETG modes present in that simulation. The GTC simulations were done with a much larger system size (thereby requiring far more computer time), and so contained lower $\mathrm{k}_{\mathrm{y}}$ modes that are more weakly affected by the noise-induced damping $\sim k_{y}^{2} D_{\text {noise. }}$ It is possible that at late times these GTC simulations had some residual ETG modes at very low $k_{y}$ that produced streamer-like structures. (Note that the contour plot of $\phi$ shown in Refs. 9 and 11 is from relatively early times in the simulation, when the observed $\chi$ is still large and the noise has not yet become dominant.) However, the fact that the PG3EQ and GTC simulations agree on the late-time transport level, when the PG3EQ simulations are clearly dominated by discrete particle noise, demonstrates that such long-wave-length streamers (if they exist) cannot have any substantial affect on the late-time electron heat transport in these GTC simulations.

Electron temperature gradient modes are seen as a candidate to explain electron heat transport across ion thermal barriers, where electron thermal conductivities of the order of $1 \mathrm{~m}^{2} / \mathrm{s}$ are often observed. In this paper we have been reporting the electron thermal conductivity in units of electron gyro-Bohms, 


$$
\chi_{e, G B}=\left(\frac{\rho_{e}}{L_{T}}\right) \rho_{e} v_{t e} \approx 0.075\left[\frac{T_{e}}{1 \mathrm{keV}}\right]^{1.5}\left[\frac{B}{1 \text { Tesla }}\right]^{-2}\left[\frac{L_{T}}{1 \mathrm{~m}}\right]^{-1} \mathrm{~m}^{2} / \mathrm{s}
$$

If $\chi_{e} \approx 13\left(\rho_{e} / L_{T}\right) \rho$ for ETG turbulence as reported in Ref. 8, this translates to $\chi_{e} \approx$ $0.98\left[\mathrm{~T}_{\mathrm{e}} / \mathrm{keV}\right]^{1.5}[\mathrm{~B} \text { Tesla }]^{-2}\left[\mathrm{~L}_{T} / \mathrm{m}\right]^{-1} \mathrm{~m}^{2} / \mathrm{s}$, a value generally consistent with experimental measurements of electron thermal transport across ion thermal barriers. Furthermore, even modest enhancements of the electron-gyro-Bohm normalized ETG transport rates, $\chi_{e} / \chi_{e, G B}$, relative to the ion-gyro-Bohm ITG transport rates, $\chi_{i} / \chi_{i, G B}$ at the same temperature gradient, can be enough to make the ETG transport experimentally relevant. This is because ion temperature gradients are often very close to marginal stability (particularly when the effects of background velocity shear and high ion to electron temperature ratio are included, which can raise the critical ion temperature gradient but have little or the opposite effect on the critical electron temperature gradient) while electron temperature gradients might exceed the ETG threshold by a large amount.

The fact that discrete particle noise has been demonstrated to be important to simulations of both ETG and ITG turbulence suggests that the PIC code development effort needs to focus on schemes for further reducing the noise level in PIC simulations. Several ideas have been proposed that may accomplish this, including the split-weight $\operatorname{algorithm~}^{17-19}$ which further reduces the perturbation in the distribution function represented by the simulation particles, and methods aimed at preventing the meansquared weight of the computer particles from growing without bound by a thermostatted $\delta f,{ }^{14}$ by periodic redistribution of the particle weights, ${ }^{15,16}$ or by periodic "regridding". ${ }^{20}$ 
However, these later methods introduce numerical dissipation, and care must be taken to ensure that this dissipation is not so large as to significantly alter the true dynamics.

In summary, we find that the conclusions of Refs. 9-11 regarding the behavior of Cyclone base-case-like ETG turbulence result from mistaking the effects of discrete particle noise for those of ETG turbulence. We conclude with the authors of Refs. 6-8 that ETG turbulence has the potential to produce substantial electron heat transport despite its high frequency and short wavelength.

\section{Acknowledgements}

We gratefully acknowledge discussions with Frank Jenko regarding his continuum ETG turbulence simulations; discussions with Mike Kotschenreuther regarding the sensitivity of ETG turbulence to noise; John Krommes for useful suggestions regarding improved forms of renormalized dielectric shielding of noise; Bruce Cohen for his interest, advice, and careful editing; and W.W. Lee and Z. Lin for their contributions to increasing interest in the subject matter addressed here. This work was performed under the auspices of the U.S. Department of Energy by UC, Lawrence Livermore National Laboratory under contract No. W-7405-ENG-48, by Princeton Plasma Physics Laboratory under contract No. DEAC02-76CH03073, and by the Center for Multiscale Plasma Dynamics at the University of Maryland under contract No. DE-FC02-04ER54784. The simulations described here 
made use of resources at the National Energy Research Supercomputer Center under Department of Energy Contract No. DE-AC03-76SF00098. 


\section{References}

1. M. Kotschenreuther, H.L. Berk, R. Denton, S. Hamaguchi, W. Horton, C-B. Kim. M. LeBrun, P. Lister, S. Mahajan, W.H. Miner, P.J. Morrison, D. Ross, T. Tajima, J.B. Taylor, P.M. Valanju, H.V. Wong, S.Y. Xiaa, and Y.-Z. Zhang, Proceedings of the $13^{\text {th }}$ International Conference on Plasma Physics and Controlled Nuclear Fusion, 1990 (IAEA, Vienna, 1991), Vol. 2, p. 361, paper IAEA-CN-53/D-IV-16.

2. A.M. Dimits and W.W. Lee, J. Comp. Phys. 107, 309 (1993); A.M. Dimits, Ph.D. thesis, Princeton University, 1988.

3. S.E. Parker and W.W. Lee, Phys. Fluids B 5, 77 (1993).

4. A.Y. Aydemir, Phys. Plasmas 1, 822 (1994).

5. G. Hu and J.A. Krommes, Phys. Plasmas 1, 863 (1994).

6. F. Jenko, W. Dorland, M. Kotschenreuther and B.N. Rogers, Phys. Plasmas 7, 1904 (May, 2000). 
7. W. Dorland, F. Jenko, M Kotschenreuther, and B.N. Rogers, Phys. Rev. Lett. 85, 5579 (December, 2000).

8. F. Jenko and W. Dorland, Phys. Rev. Lett. 89, 225001 (November, 2002).

9. Z. Lin, L. Chen, Y. Nishimura, H. Qu, T.S. Hahm, J.L.V. Lewandowski, G. Rewoldt, W.X. Wang, P.H. Diamond, C. Holland, F. Zonca, and Y. Li, Electron Thermal transport in Tokamaks: ETG or TEM Turbulence? Fusion Energy 2004, Proc. $20^{\text {th }}$ Fusion Energy Conference, Vilamoura, 2004 (IAEA, Vienna, 2005) paper TH8_4. See http://www.cfn.ist.utl.pt/20IAEAConf/presentations/T5/2T/5_H_8_4/Talk_TH_8_4.pdf and http://physics.ucsd.edu/plasmatheorygroup/LINiaea04.pdf.

10. Z. Lin, On Structures in Electron Temperature Gradient Turbulence, Bull. Am. Phys. Soc. (November, 2004). See http://www.aps.org/meet/DPP04/baps/index.html .

11. Z. Lin, L. Chen, and F. Zonca, Phys. Plasmas 12, 056125 (May, 2005).

12. A.M. Dimits, G. Bateman, M.A. Beer, B.I. Cohen, W. Dorland, G.W. Hammett, C. Kim, J.E. Kinsey, M. Kotschenreuther, A.H. Kritz, L.L. Lao, J. Mandrekas, W.M. Nevins, S.E. Parker, A.J. Redd, D.E. Shumaker, R. Sydora, and J. Weiland, Phys. Plasmas 7, 969 (March, 2000). 
13. A.M. Dimits, , T.J. Williams, J.A. Byers, and B.I. Cohen, Phys. Rev. Lett. 77, 71 (1996).

14. J.A. Krommes, Phys. Plasmas 6, 1477 (May, 1999).

15. S. Brunner, E. Valeo, and J.A. Krommes, Phys. Plasmas 6, 4505 (December, 1999).

16. W.X. Wang, N. Nakajima, M. Okamoto, and S. Murakami, Plasma Phys. Controlled Fusion 41, 1091 (1999).

17. I. Manuilskiy, and W.W. Lee, Phys. Plasmas 7, 2933 (July, 2000).

18. W.W. Lee, J.L.V. Lewandowski, T.S. Hahm, and Z. Lin, Phys. Plasmas 8, 4435 (October, 2001)

19. J.L.V. Lewandowski, Phys. Plasmas 10, 3204 (August, 2003).

20. S. Vadlamani, S. E. Parker, Y. Chen, and C. Kim, Comp. Phys. Comm. 164, 209 (2004).

21. W.W. Lee, Steady State Global Simulations of Microturbulence, Bull. Am. Phys. Soc. (November, 2004). See http://www.aps.org/meet/DPP04/baps/index.html . 
22. A. Bruce Langdon, Phys. Fluids 22, 163 (January, 1979).

23. C.K. Birdsall and A.B. Langdon, Plasma Physics via Computer Simulation, (McGraw-Hill, New York, 1985).

24. J.A. Krommes, Phys. Fluids B 5, 1066 (April, 1993).

25. G. Hammett, private communication (2005). See also, G. W. Hammett, A.M. Dimits, and W.M. Nevins, 2005 International Sherwood Fusion Theory Conference (Incline Village, Nevada, 2005), https://wormhole.ucllnl.org/sherwood05/absindex.html\#O3 .

26. P.J. Roache, Verification and Validation in Computational Science and Engineering, (Hermosa Publishers, Albuquerque, NM, 1998).

27. W.W. Lee, Phys. Fluids 26, 556 (February, 1983).

28. W.W. Lee and W. Tang, Phys. Fluids 31, 612 (1988).

29. C. Bolton, private communication (April, 2005).

30. Z. Lin, private communication (Sherwood Int. Fusion Theory Conf., April, 2005). 
31. M. Kotschenreuther, G. Rewoldt, and W.M. Tang, Comp. Phys. Comm. 88, 128 (1995).

32. P.J. Catto, Phys. Fluids 21, 147 (1978).

33. J.A. Krommes, "Fundamental Statistical Descriptions of Plasma Turbulence in Magnetic Fields", Physics Reports 360, 1 (2002). 


\section{Figure Captions}

Figure 1. (Color online) The linear growth rate in units of $\left(v_{t} / L_{T}\right)$ for Cyclone base-caselike ETG modes is plotted vs. the wave number in the bi-normal direction, both with (red curve) and without (green curve) magnetically trapped electrons. For comparison, we also plot an estimate of the damping rate, $k_{y}^{2} D$, that would be associated with noiseinduced diffusion for $D=0.5\left(\rho / L_{T}\right) \rho v_{t}$ (blue curve) or $D=2.0\left(\rho / L_{T}\right) \rho v_{t}$ (purple curve)).

Figure 2. (Color online) The coefficient of electron thermal transport, $\chi_{e}(t)$, from a particle-number and box-size convergence study of Cyclone base-case-like ETG turbulence without magnetic trapping $\left(r / R_{0}=0\right)$ including runs in with a flux-tube crosssection of $125 \rho_{e} \times 125 \rho_{e}$ and 2 particles/grid cell (blue curve), 4 particles/grid cell (green curve), and 8 particles/grid cell (black curve); and a flux-tube cross-section of $250 \rho_{e} \times 62.5 \rho_{e}$ with 16 particles/grid cell (red curve).

Figure 3. (Color online) The ETG fluctuation spectra in the linear phase, $t=420 L_{T} / v_{t e}$ ( $\mathrm{D}_{420}$, red curve) and at $t \approx 20 / \gamma_{0}$ after saturation from the PG3EQ simulation with a crosssection of $125 \rho_{e} \times 125 \rho_{e}$ and 8 particles/grid cell ( D $995_{9}$, black curve) corresponding to the black curve in Fig. 2. 
Figure 4. (Color online) The coefficient of electron thermal transport, $\chi_{e}(t)$, from a particle-number and flux tube cross-section convergence study of Cyclone base-case-like ETG turbulence with magnetic trapping $\left(r / R_{0}=0.18\right)$, including runs in a flux-tube with cross-section $500 \rho_{e} \times 125 \rho_{e}$ with 2 particles/grid cell (green curve), 4 particles/grid cell (blue curve), and 16 particles/grid cell (red curve); and a flux-tube cross-section of $250 \rho_{e} \times 62.5 \rho_{e}$ with 16 particles/grid cell (black curve).

Figure 5 (Color online) The ETG fluctuation spectra in the linear phase, $t=400 L_{T} / v_{t e}$ $\left(\mathrm{D}_{400}\right.$, red curve) and at 30 linear growth times later at $t \approx 1228 L_{T} / v_{t e}\left(\mathrm{D}_{1228}\right.$, black curve $)$. Data from the PG3EQ simulation with a cross-section of $250 \rho_{e} \times 62.5 \rho_{e}$ and 16 particles/grid cell (corresponding to the black curve in Fig. 4).

Figure 6. Grey-tone rendering of the potential on the outboard mid-plane at $t=400 L_{T} / v_{t e}$ late in the linear phase (top panel) and 30 linear growth times later at $t=1228 L_{T} / v_{\text {te }}$ (middle panel) show the characteristic ETG "streamers". These streamers are absent at very late times, $t=2500 L_{T} / v_{t e}$ (bottom panel) during the steady-state phase of the simulation. Data from the PG3EQ simulation of Cyclone base-case-like ETG turbulence with flux-tube cross-section $250 \rho_{e} \times 62.5 \rho_{e}$, magnetic trapping $\left(r / R_{0}=0.18\right)$, and 16 particles/grid cell (black curve of Fig. 4). 
Figure 7. (Color online) (a) The fluctuation spectrum at the outboard mid-plane averaged over the radial coordinate $(x)$ and the interval $2500 L_{T} / v_{t e}<t<3000 L_{T} / v_{t e}$ is plotted on a semi-log scale vs. $k_{y}$ ( $\mathrm{D}_{\text {late, }}$ black curve), together with the corresponding fully uncorrelated noise estimate $(\mathrm{N}$, blue curve) and self-Debye shield noise estimate $(\mathrm{H}$, red curve). The fluctuation spectrum averaged over radius and the interval $1200 L_{T} / v_{t e}<$ $t<1300 L_{T} / v_{t e}\left(\mathrm{D}_{\text {early, }}\right.$ green curve) and the corresponding self-Debye shielded noise level ( $\mathrm{H}_{\text {early }}$ chartreuse curve) are shown for comparison. (b) Fluctuation and noise data for the interval $2500 L_{T} / v_{t e}<t<3000 L_{T} / v_{t e}$ on a linear scale.

Figure 8. (Color online) (a) The fluctuation spectrum at the outboard mid-plane averaged over the bi-normal coordinate $(y)$ and the interval $2500 L_{T} / v_{t e}<t<3000 L_{T} / v_{t e}$ is plotted on a semi-log scale vs. $k_{x}$ (D, black curve), together with the corresponding fully uncorrelated noise estimate $(\mathrm{N}$, blue curve) and self-Debye shield noise estimate $(\mathrm{H}$, red curve). (b) Same data on a linear scale.

Figure 9. (Color online) The fluctuation energy averaged over the outboard mid-plane (black curve) is compared to the fluctuation intensity from the fully uncorrelated noise spectrum (blue curve) or self-Debye shielded noise spectrum (red curve). 
Figure 10. (Color online) The fluctuation energy is plotted vs. time for a PG3EQ simulation of Cyclone base-case ITG turbulence (black curve). The red curve shows the fluctuation energy expected from the discrete particle noise. The corresponding level of thermal transport from this PG3EQ simulation is shown by the blue curve.

Figure 11. (Color online) Electron heat flux from the "Noise Test" of Lin and Bolton. The black curve is from the initial simulation. The remaining 5 curves correspond to simulations initialized with $\left\langle w^{2}\right\rangle_{\text {initial }}=\left\langle w^{2}\right\rangle_{\text {final }}($ red curve $),\left\langle w^{2}\right\rangle_{\text {initial }}=(1 / 2)\left\langle w^{2}\right\rangle_{\text {final }}$ (blue curve), $\left\langle w^{2}\right\rangle_{\text {initial }}=(1 / 4)\left\langle w^{2}\right\rangle_{\text {final }}$ (gold curve), $\left\langle w^{2}\right\rangle_{\text {initial }}=(1 / 8)\left\langle w^{2}\right\rangle_{\text {final }}$ (green curve), $\left\langle w^{2}\right\rangle_{\text {initial }}=(1 / 32)\left\langle w^{2}\right\rangle_{\text {final }}$ (chartreuse curve).

Figure 12. (Color online) (a) the intensity of the dominant Fourier mode during the linear phase of each run in Fig. 11. The black curve is $k_{y} \rho_{e}=0.3$ from the initial simulation (multiplied by $10^{6}$ ). In the remaining 5 curves $t$ is measured from the time of the restart. For $\left\langle w^{2}\right\rangle_{\text {initial }}=\left\langle w^{2}\right\rangle_{\text {final }}\left(\right.$ red curve) and $\left\langle w^{2}\right\rangle_{\text {initial }}=(1 / 2)\left\langle w^{2}\right\rangle_{\text {final }}$ (blue curve) the dominant mode is $k_{y} \rho_{e}=0.1$. For $\left\langle w^{2}\right\rangle_{\text {initial }}=(1 / 4)\left\langle w^{2}\right\rangle_{\text {final }}$ (gold curve), $\left\langle w^{2}\right\rangle_{\text {initial }}=(1 / 8)$ $\left\langle w^{2}\right\rangle_{\text {final }}$ (green curve), and $\left\langle w^{2}\right\rangle_{\text {initial }}=(1 / 32)\left\langle w^{2}\right\rangle_{\text {final }}$ (chartreuse curve) the dominant mode is $k_{y} \rho_{e}=0.2$. (b) The same data re-plotted to display the weak linear growth of the $k_{y} \rho_{e}=0.1$ mode in the restart with $\left\langle w^{2}\right\rangle_{\text {initial }}=(1 / 2)\left\langle w^{2}\right\rangle_{\text {final }}$ (blue curve). 
Figure 13. (Color online) The real frequency (a) and growth rate (b) for Cyclone basecase-like ETG turbulence as a function of the magnitude of the diffusion, $D_{\text {noise }}$ acting on the non-adiabatic part of the electron distribution function for $k_{y} \rho_{e}=0.1$ (black curves), $k_{y} \rho_{e}=0.2$ (red curves), $k_{y} \rho_{e}=0.3$ (blue curves), $k_{y} \rho_{e}=0.4$ (green curves), and $k_{y} \rho_{e}=0.5$ (chartreuse curves).

Figure 14. (Color online) Measured maximum linear growth rate (with error bars and connected by the grey line) after restart from the noise test simulations of Sec. IV are compared with $\gamma\left(k_{y}, D_{\text {noise }}\right)$ from Eq. 15 with $\alpha_{\text {noise }} \approx 1$ (heavy line). The colors of both data points and model are chosen to indicate the corresponding wave number as $k_{y} \rho_{e}=0.1$ (black), $k_{y} \rho_{e}=0.2$ (red), and $k_{y} \rho_{e}=0.3$ (blue).

Figure 15. (Color online) The value of $\chi_{\text {noise }}(t)$ from Eq. (16) for Cyclone base-case-like ETG turbulence with magnetic trapping $\left(r / R_{0}=0.18\right)$ from PIC simulation in a flux-tube cross-section of $250 \rho_{e} \times 62.5 \rho_{e}$ with 16 particles/grid cell is displayed both with (red curve) and without (black curve) the contribution of turbulence, toroidal drifts, and magnetic shear to the decorrelation. 
Figure 16. The predicted value of $\chi_{\text {noise }}(t)=(3 / 2) D_{\text {noise }}(t)$ from Eq. 16 is compared to $\chi_{e}(t)$ from noise test described in Sec. IV. The black curves are $\chi_{e}(t)$ from the initial simulation (distinguished by large variations) and from Eq. 16 (smooth curve at $\chi \approx 3$ ). The remaining curves show simulations initialized with $\left\langle w^{2}\right\rangle_{\text {initial }}=\left\langle w^{2}\right\rangle_{\text {final }}$ (red curves), $\left\langle w^{2}\right\rangle_{\text {initial }}=(1 / 2)\left\langle w^{2}\right\rangle_{\text {final }}$ (blue curves), $\left\langle w^{2}\right\rangle_{\text {initial }}=(1 / 4)\left\langle w^{2}\right\rangle_{\text {final }}$ (gold curves), $\left\langle w^{2}\right\rangle_{\text {initial }}=(1 / 8)$ $\left\langle w^{2}\right\rangle_{\text {final }}$ (green curves), $\left\langle w^{2}\right\rangle_{\text {initial }}=(1 / 32)\left\langle w^{2}\right\rangle_{\text {final }}$ (chartreuse curves).

Figure 17. (Color online) (a) The $\chi_{e}(t)$ from noise test described in Sec. IV (larger variation), and the predictions from Eq. 16 (smaller variation), with $\left\langle w^{2}\right\rangle_{\text {initial }}=16\left\langle w^{2}\right\rangle_{\text {final }}$ (black curves), $\left\langle w^{2}\right\rangle_{\text {initial }}=4\left\langle w^{2}\right\rangle_{\text {final }}$ (blue curves), $\left\langle w^{2}\right\rangle_{\text {initial }}=\left\langle w^{2}\right\rangle_{\text {final }}$ (red curves). (b) The predicted value of $\chi_{\text {noise }}$ from Eq. 16 (green squares) and the simulation results (red crosses) from all of the random restart tests in Figs. 16 and 17(a), showing that Eq.16 predicts the observed scaling well as the average squared weight is varied by a factor of 512.

Figure 18. (Color online) (a) The net linear growth rate vs. time $\left(\alpha_{\text {noise }}=1.0\right)$ for $k_{y} \rho_{e}=0.1$ (black curves), $k_{y} \rho_{e}=0.2$ (red curves), and $k_{y} \rho_{e}=0.3$ (blue curves). The thick (upper) curves use $D_{\text {noise }}$ from Eq. 16 . The thin (lower) curves show the sensitivity to turbulent de-correlation by setting $v_{\text {turb }}=0$. (b) The intensity of Fourier modes vs. time for $k_{y} \rho_{e}=0.1$ (black curve), $k_{y} \rho_{e}=0.2$ (red curve), $k_{y} \rho_{e}=0.3$ (blue curve), $k_{y} \rho_{e}=0.4$ (green curve), and $k_{y} \rho_{e}=0.5$ (chartreuse curve). 


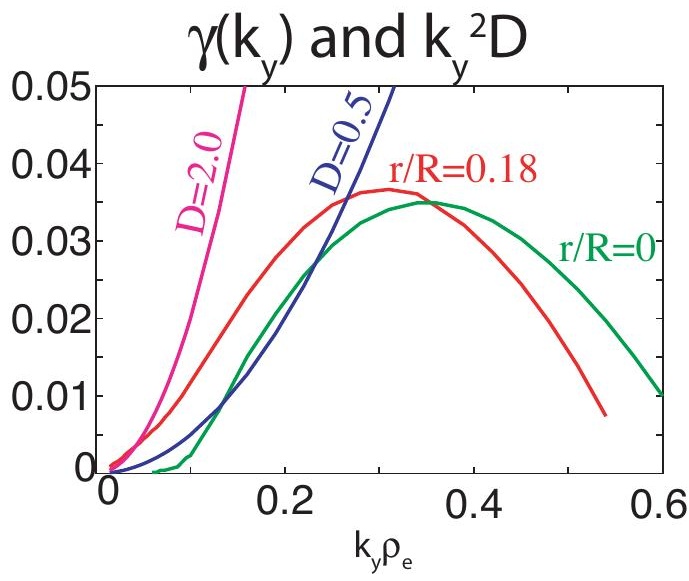


$\chi_{e}[t]\left(\left(\rho_{e} / L_{T}\right) \rho_{e} v_{t h}\right)$

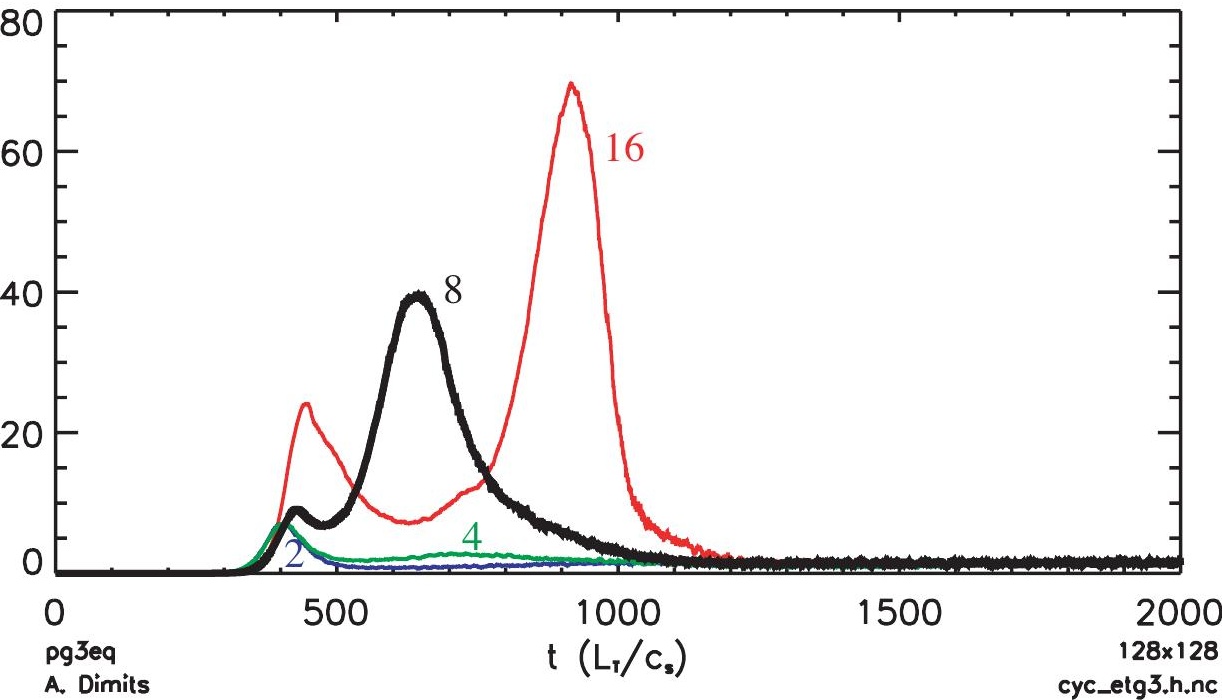


$\int|\phi|^{2} \mathrm{dk}_{\mathrm{x}}\left[\mathrm{k}_{\mathrm{y}}, \mathrm{t}=995.\right]$

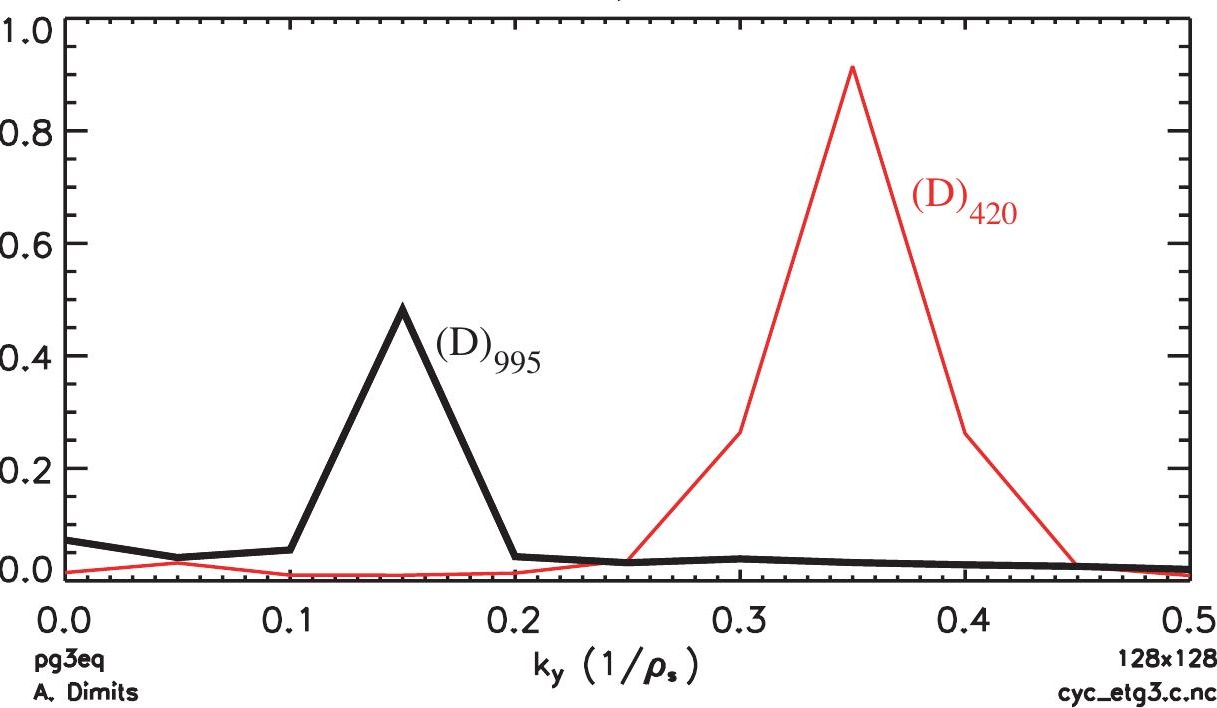


$\int|\phi|^{2} \mathrm{dk}_{\mathrm{x}}\left[\mathrm{k}_{y}, \mathrm{t}=1.23 \mathrm{E}+03\right]$

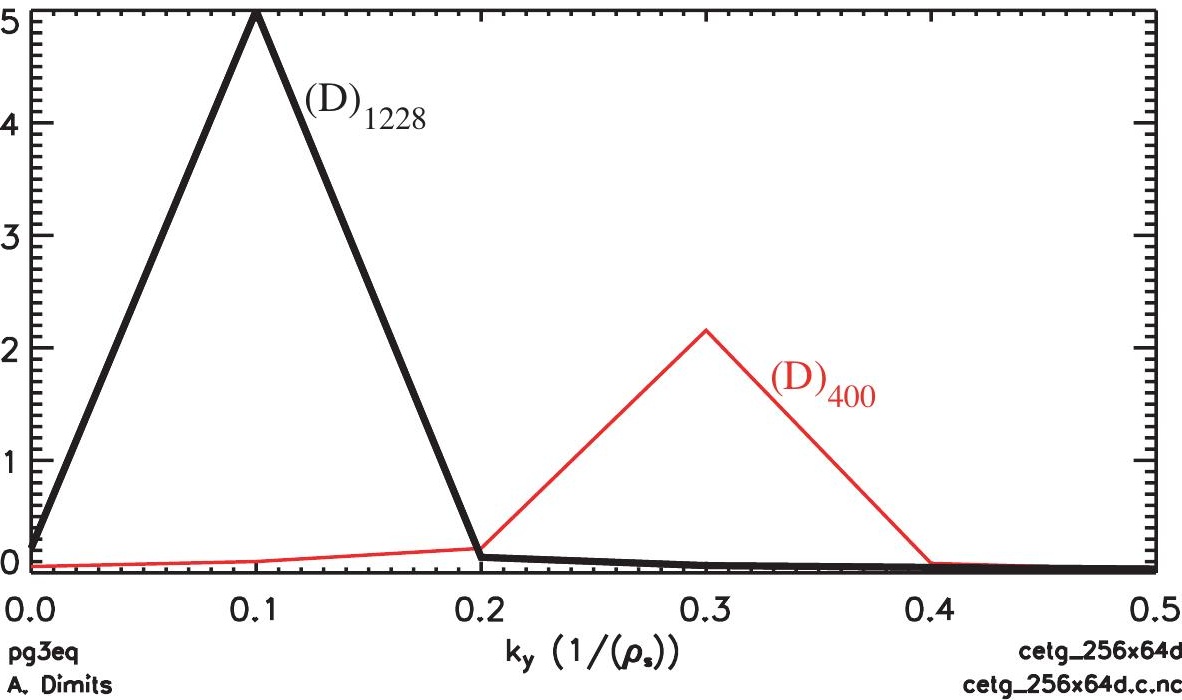


$\left\langle\Sigma_{\mathrm{ky}}|\phi|^{2}\right\rangle_{\mathrm{l}}\left[\mathrm{k}_{\mathrm{x}}\right]$

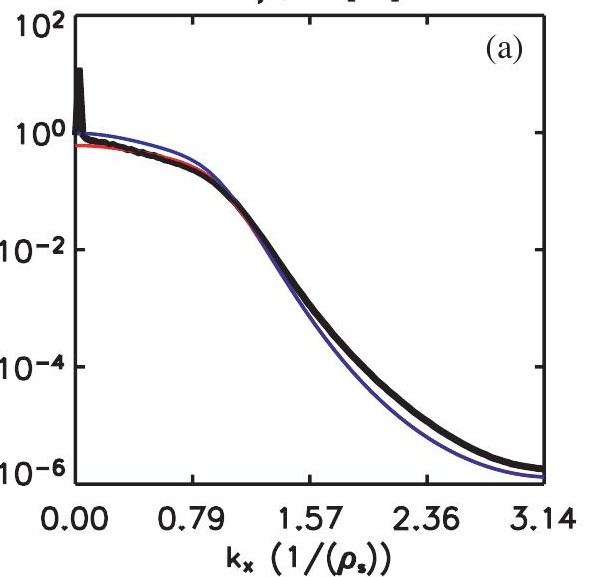

$\left\langle\Sigma_{\mathrm{ky}}|\phi|^{2}\right\rangle_{\mathrm{t}}\left[\mathrm{k}_{\mathrm{x}}\right]$

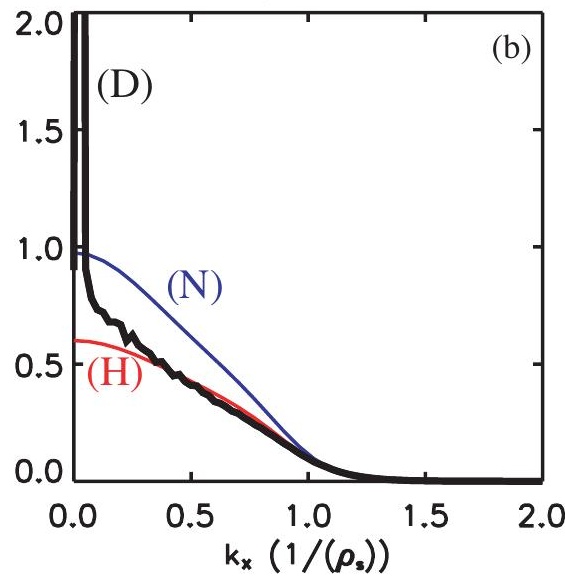


$\varepsilon_{\phi}[t]\left(\left(\rho / L_{T}\right)^{2} n T\right)$

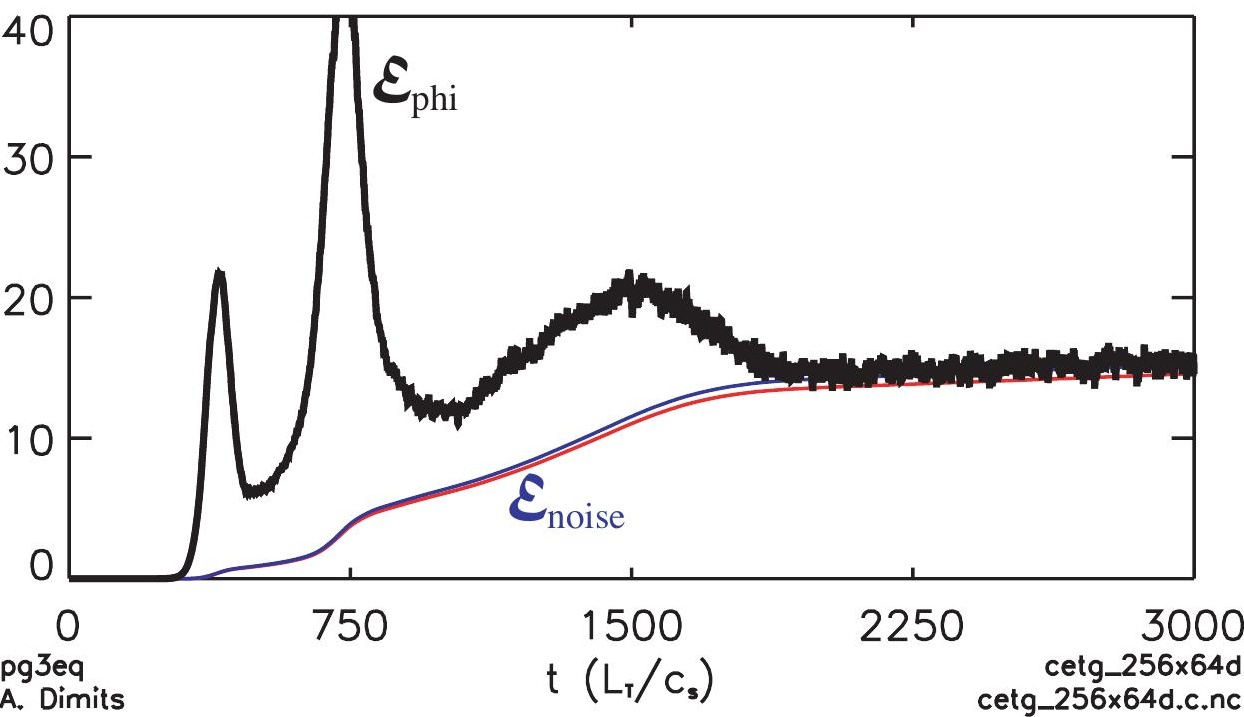


$\chi_{e}[t]\left(\left(\rho_{e} / L_{T}\right) \rho_{e} v_{t e}\right)$

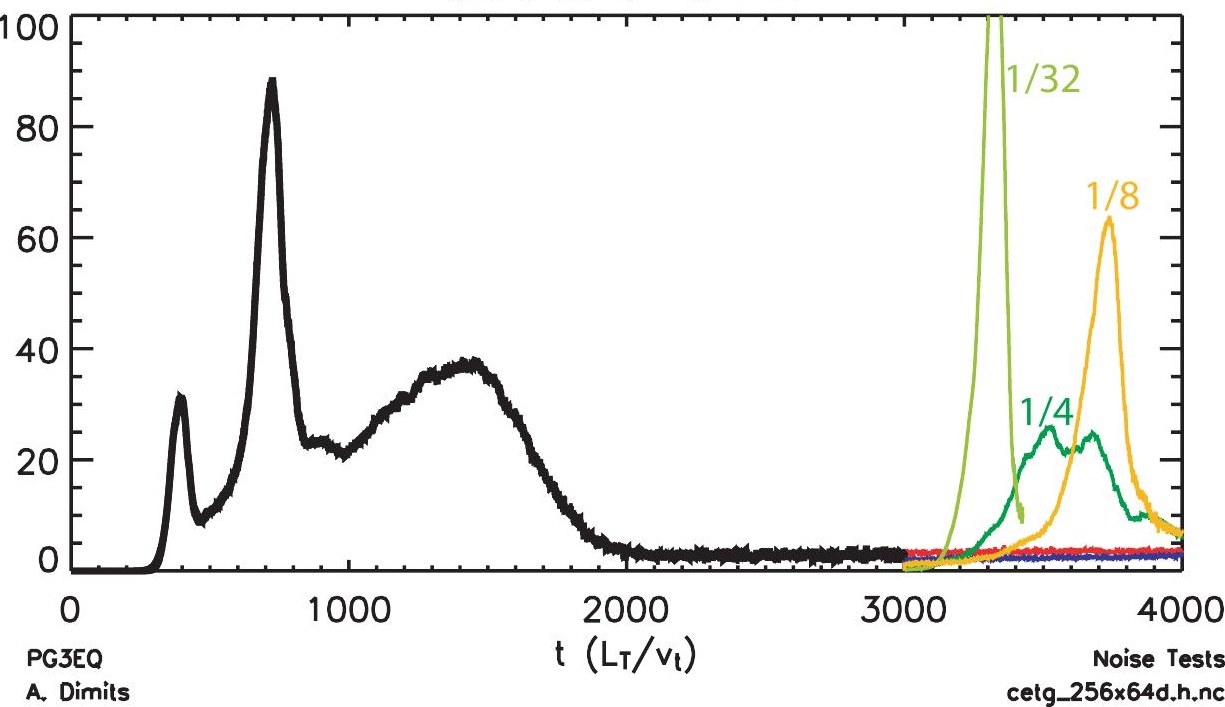


$\gamma_{\max }\left(v_{t e} / L_{T}\right)$

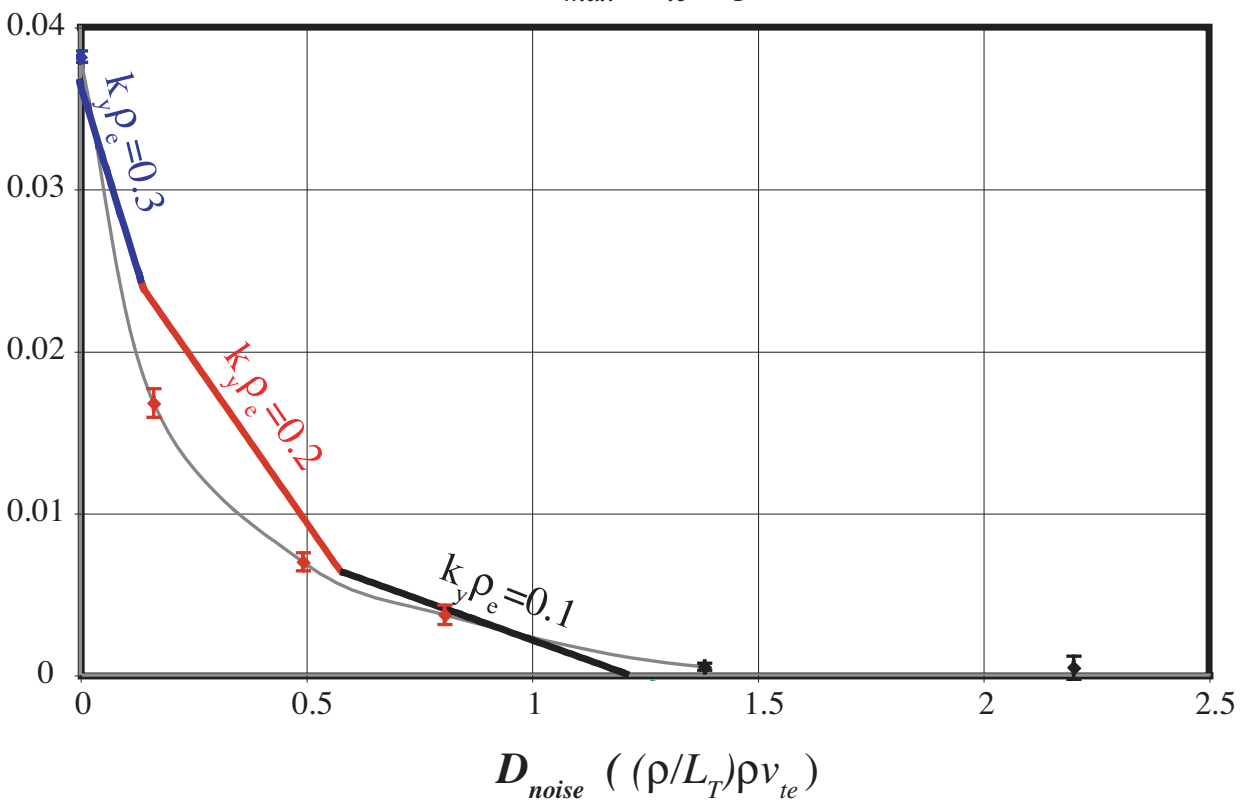


$\chi_{\mathrm{e}}[\mathrm{t}]\left(\left(\rho / \mathrm{L}_{\mathrm{T}}\right) \rho v_{\mathrm{t}}\right)$

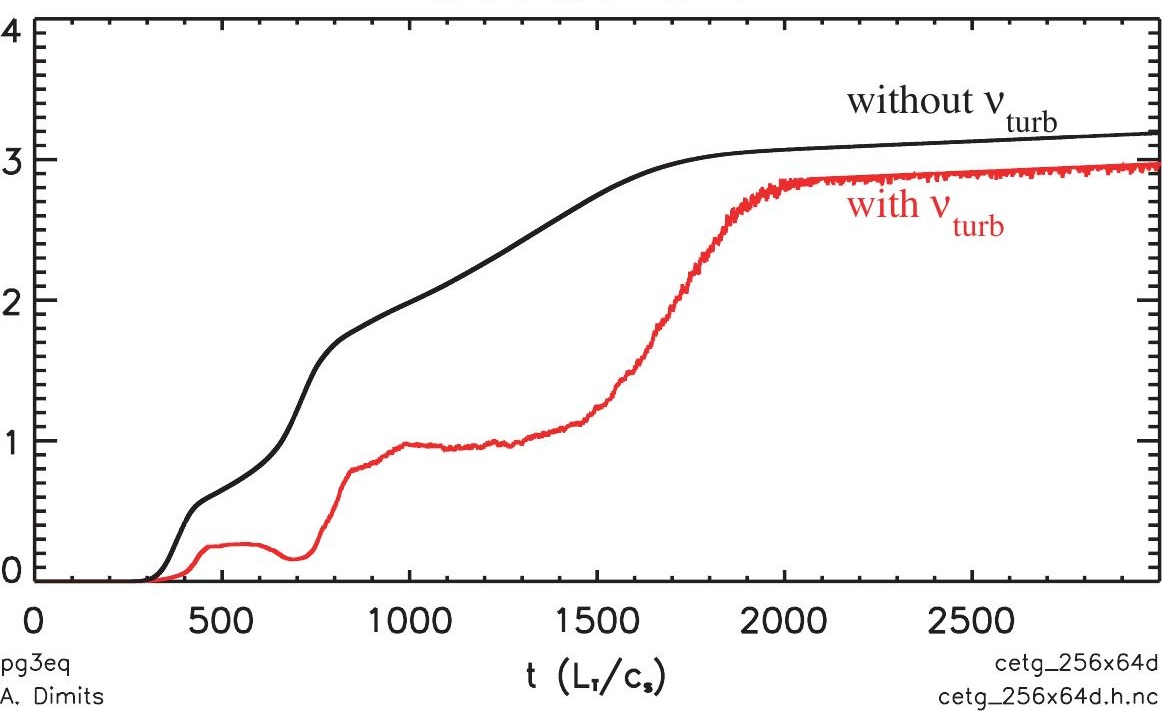


$\gamma\left[k_{y}, t\right]\left(v_{t} / L_{T}\right)$

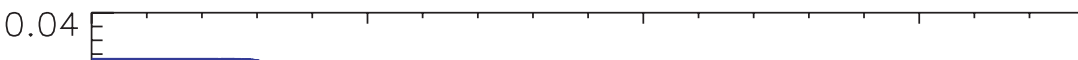

(a)

0.03

$\mathrm{k}_{\mathrm{y}} \rho_{\mathrm{e}}=0.3$

$0.01=\mathrm{k}_{\mathrm{y}} \rho_{\mathrm{e}}=0.1$

0.00 $\mathrm{k}_{\mathrm{y}} \rho_{\mathrm{e}}=0.2$

pg3eq

A. Dimits

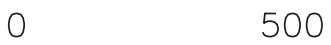

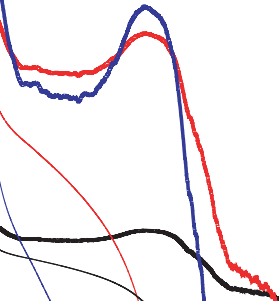

400

200

$\sum_{\mathrm{kx}}|\phi|^{2}\left[\mathrm{k}_{\mathrm{y}}, \mathrm{t}\right]$

cetg_256×64d cetg_256x64d.h.nc pg3eq

A. Dimits

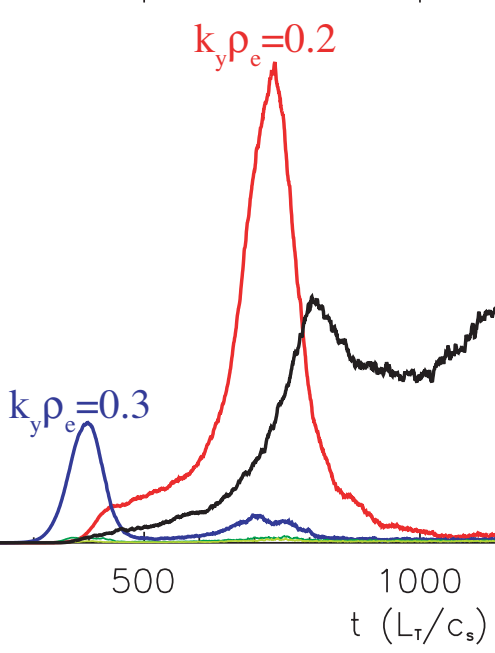

t $\left(L_{T} / C_{S}\right)$

(b)

0 